\title{
PEPS as ground states: degeneracy and topology
}

\author{
Norbert Schuch, ${ }^{1,2}$ Ignacio Cirac, ${ }^{2}$ and David Pérez-García ${ }^{3}$ \\ 1 California Institute of Technology, Institute for Quantum Information, \\ MC 305-16, Pasadena CA 91125, USA \\ 2 Max-Planck-Institut für Quantenoptik, \\ Hans-Kopfermann-Str. 1, D-85748 Garching, Germany \\ 3 Dpto. Analisis Matematico and IMI, \\ Universidad Complutense de Madrid, 28040 Madrid, Spain
}

\begin{abstract}
We introduce a framework for characterizing Matrix Product States (MPS) and Projected Entangled Pair States (PEPS) in terms of symmetries. This allows us to understand how PEPS appear as ground states of local Hamiltonians with finitely degenerate ground states and to characterize the ground state subspace. Subsequently, we apply our framework to show how the topological properties of these ground states can be explained solely from the symmetry: We prove that ground states are locally indistinguishable and can be transformed into each other by acting on a restricted region, we explain the origin of the topological entropy, and we discuss how to renormalize these states based on their symmetries. Finally, we show how the anyonic character of excitations can be understood as a consequence of the underlying symmetries.
\end{abstract}

\section{Introduction}

What are the entanglement properties of quantum many-body states which characterize ground states of Hamiltonians with local interactions? The answer seems to be "an area law": the bipartite entanglement between any region and its complement grows as the area separating them - and not as their volume, as is the case for a random state (see [1] for a recent review). Moreover, particular corrections to this scaling law are linked with critical points (logarithmic corrections) or topological order (additive corrections). A rigorous general proof of the area law, however, could up to now only be given for the case of onedimensional systems 2, where an area law has been proven for all systems with an energy gap above the ground state, whereas the currently strongest result for two dimensions 3, 3, requires a hypothesis on the eigenvalue distribution of the Hamiltonian. Surprisingly, there is a completely general proof in arbitrary dimensions if instead, we consider the corresponding quantity for thermal states [5, and similar links to topological order persist [6]. 
The area law can be taken as a guideline for designing classes of quantum states which allow to faithfully approximate ground states of local Hamiltonians. There are several of these classes in the literature: Matrix Product States (MPS) [7] and Projected Entangled Pair States (PEPS) [8] are most directly motivated by the area law, but there are other approaches such as MERA (the Multi-Scale Entanglement Renormalization Ansatz) 9 which e.g. is based on the scale invariance of critical systems; all these classes are summarized under the name of Tensor Network or Tensor Product States. Though the main motivation to introduce them was numerical - they consitute variational ansatzes over which one minimizes the energy of a target Hamiltonians and thus obtains an approximate description of the ground state - they have turned out to be powerful tools for characterizing the role of entanglement in quantum many body systems, and thus helped to improve our understanding of their physics.

In this paper, we are going to present a theoretical framework which allows us to understand how MPS and PEPS appear as ground states of local Hamiltonians, and to characterize the properties of their ground state subspace. This encompasses previously known results for MPS and particular instances of PEPS, while simultaneously giving rise to a range of new phenomena, in particular topological effects. Our work is motivated by the contrast between the rather complete understanding in one and the rather sparse picture in two dimensions, and we will review what is known in the following. We will thereby focus on analytical results, and refer the reader interested in numerical aspects to 10 .

\subsection{Matrix Product States}

Matrix Product States (MPS) 7] form a family of one-dimensional quantum states whose description is inherently local, in the sense that the degree to which two spins can be correlated is related to their distance. The total amount of correlations across any cut is controlled by a parameter called the bond dimension, such that increasing the bond dimension allows to grow the set of states described. MPS have a long history, which was renewed in 1992 when two apparently independent papers appeared: In 11], Fannes, Nachtergaele, and Werner generalized the AKLT construction of 12 by introducing the so-called Finitely Correlated States, which in retrospect can be interpreted as MPS defined on an infinite chain; in fact, this work layed the basis for our understanding of MPS and introduced many techniques which later proved useful in characterizing MPS [13. The other was 14], where White introduced the Density Matrix Renormalization Group (DMRG) algorithm, which can now be understood as a variational algorithm over the set of MPS. In [15], MPS were explained from a quantum information point of view by distributing "virtual" maximally entangled pairs between adjacent sites which can only be partially accessed by

acting on the physical system. This entanglement-based perspective has since then fostered a wide variety of results. 


\section{The complexity of simulating $1 \mathrm{D}$ systems}

Motivated by the extreme success of DMRG, people investigated how hard or easy the problem of approximating the ground state of a 1D local Hamiltonian (or simply its energy) was. The history of this problem is full of interesting positive and negative results. A number of them was devoted to prove that every ground state of a gapped 1D local Hamiltonian can be approximated by an MPS 16, 17; this was finally proven by Hastings 2, justifying the use of MPS as the appropriate representation of the state of one-dimensional spin systems. Very recently, also in the positive, it was shown that dynamical programming could be used to find the best approximation to the ground state of a one-dimensional system within the set of MPS with fixed bond dimension in a provably efficient way $[18,19$. On the other hand, in the negative it could be shown that finding the ground state energy of Hamiltonians whose ground states are MPS with a bond dimension polynomial in the system size is NP-hard [20]; this is based on a previous result of Aharonov et al. 21 proving that finding the ground state energy of 1D Hamiltonians is QMA-complete (the quantum version of NP-complete).

\section{Hidden orders, symmetries and entanglement in spin chains}

As we have seen, MPS provide the right description for one-dimensional quantum spin chains. Therefore, and given their simple structure, one can employ MPS to improve our understanding of the physics of one-dimensional systems. One field in which significant insight could be gained was the characterization of symmetries in terms of entanglement. First, the relation between string order parameters and localizable entanglement was explained in 22, 23. In 24] (see also 25]), global symmetries in generic MPS have been characterized, and related to the existence of string order parameters, thus explaining many of the properties of string order, for instance its fragility 26. This characterization of global symmetries was generalized to arbitrary MPS in [27, where it was used to shed light on the Hamiltonian-free nature of the Lieb-Schultz-Mattis theorem as well as to find new $\mathrm{SU}(2)$-invariant two-body Hamiltonians with MPS ground states, beyond the AKLT and Majumdar-Ghosh models. Other examples of MPS with global symmetries were already provided in $11,28,29$. Recently, also reflection symmetry has been investigated, showing how it provides topological protection of some MPS such as the odd-spin AKLT model, as opposed to the even-spin case [30].

MPS have also been extremely useful in understanding the scaling of entanglement in quantum spin chains, where special attention has been devoted to the case of quantum phase transitions. In [31, MPS were used to give examples of phase transitions with unexpected properties, namely analytic ground state energy and finite entanglement entropy of an infinite half-chain; the entanglement properties of these examples were further analyzed in [32 33. In [34], MPS theory was used to compute how the geometric entanglement with respect to large blocks diverges logarithmically with the correlation length near a critical 
point, and thus takes the role of an order parameter (see also 33]).

Apart from that, MPS theory has been used to decompose global operations (such as cloning or the creation of an entangled state) into a sequence of local operations 35, 36], to characterize renormalization group transformations and their fixed points in 1D [37, to understand which quantum circuits can be simulated classically 38 , or even to propose new numerical methods to solve differential equations [39] or to compress images [40].

But what is it that makes MPS so useful in deriving all these results?

\section{The structure of MPS}

The main reason seems to be that MPS, despite being able to faithfully represent the states of one-dimensional systems, have a simple and well-understood structure, which makes them quite easy to deal with. For instance, as shown in [38, they naturally reflect the Schmidt decomposition at any cut across the chain, which makes dealing with their entanglement properties particularly easy. If one moreover restricts to the physically relevant case of translational invariant states, it turns out that one can fully characterize the set of all translationally invariant MPS by bringing them into a canonical form [11.13]; in fact this canonical form constitutes one of the main ingredients in many of the results mentioned above.

In the canonical form, the matrices characterizing the MPS obtain a block diagonal form, and the properties of the state can be simply read off the structure of these blocks. In particular, for the case of one block (termed the injective case), it can be shown that the MPS arises as the unique ground state of a so-called "parent" Hamiltonian with local interactions, which moreover is frustration free. For the non-injective case where one has several blocks, the number of blocks determines the degeneracy of the parent Hamiltonian, and the ground state subspace is spanned by the injective MPS described by the individual blocks 13. Beyond that, the injective case has other nice properties, such as an exponential decay of correlations. For the case of an infinite chain, all these properties were proven in [11], together with the fact that the parent Hamiltonian of an injective MPS has an energy gap above the ground state. The block structure of the canonical form is also useful beyond the relation of Hamiltonians and ground states, and e.g. allows to read off the type of the RG fixed point of a given 1D system.

\section{$1.2 \quad$ PEPS}

Projected Entangled Pair States (PEPS) constitute the natural generalization of MPS to two and higher dimensions, motivated by the quantum information perspective on MPS which views them as arising from virtual entangled pairs between nearest neighbors [8, 41]. Though there has not yet been a complete formal proof that PEPS approximate efficiently all ground states of gapped local Hamiltonians, this could be proven under a (realistic) assumption on the spectral density in the low-energy regime [42], showing that PEPS are the appropriate 
class to describe a large variety of two-dimensional systems. However, as compared to MPS, PEPS are much harder to deal with: For instance, computing expectation values of local observables, which would be the key ingredient in any variational algorithm such as DMRG, is a \#P-complete problem, and thus in particular NP-hard [43]. This poses an obstacle to numerical methods, and different ideas to overcome this problem have been proposed (we refer again to [10] for numerical issues). Fortunately, the bad news comes with good ones: The increase in complexity allows to find a much larger variety of different interesting behavior within PEPS as compared to MPS; for instance, in [44 it is shown that there exist PEPS with a power-law decay of two-point correlation functions, something which cannot be achieved for MPS.

\section{Many examples}

There have been identified various different classes of PEPS which exhibit rich properties. To start with, in 45 it has been shown how the interpretation of the $2 \mathrm{D}$ cluster state as a PEPS can be used to understand measurement based quantum computation [46] - a way of performing a quantum computation solely by measurements - by viewing it as a way to carry out the computation as teleportation-based computation on the virtal maximally entangled states underlying the PEPS description. This motivated the search for different models for measurement based quantum computation, and indeed models with very different properties have been subsequently proposed $47-49$.

Another category of examples has been found with regard to topological models, where it was realized that many topologically ordered states have a PEPS representation with a small bond dimension: The case of Kitaev's toric code was observed in [4, and this was later generalized to all string net models in 50. Yet, despite their ability to describe these states, up to now PEPS did not help much in understanding the topological behavior of these states, which is one of the things we will assess in this work.

\section{Few general results}

As we have seen, the class of PEPS is rich enough to incorporate states with a variety of different behaviors. However, given the complexity of topological systems or of (measurement based) quantum computation, is this class still simple enough to prove useful as a tool to improve our understanding of $2 \mathrm{D}$ systems? That is, can PEPS help to uncover new effects and relations in nature, or to give a better understanding of the mechanisms behind quantum effects in two dimensions?

Judging from the experience with one-dimensional systems, in order to do so it would be highly desirable to have an understanding of the structure of PEPS comparable to the one obtained using the canonical form in one dimension. As it turns out, in the case of "injective" PEPS, several 1D results can be transferred; in particular, injective PEPS appear as unique ground states of their parent Hamiltonian 51. Also, in 52] it is shown how global symmetries can 
be characterized in injective PEPS, which helped to understand the mechanism behind the 2D version of the Lieb-Schultz-Mattis theorem [53], to define an appropriate analogue for string orders in 2D 24], and to improve the PEPSbased algorithms used to simulate 2D systems with symmetries [54. While these results illustrate that PEPS are a useful tool to understand properties of quantum states which appear as unique ground states of local Hamiltonians, it is also true that some of the most interesting physics in two dimensions takes place in systems which do not have unique ground states, but rather lowly degenerate ones, such as systems with symmetry broken phases or states with topological order.

Yet, in order to be able to fully apply the toolbox of PEPS to the understanding of these systems, it would be crucial to have a mathematical characterization of the structure of PEPS, providing a framework similar to the one which proved so useful for one-dimensional systems: Is there a canonical form for PEPS which allows to easily determine their properties, and how can it be found? How do PEPS appear as ground states of local "parent" Hamiltonians, and what is the ground state degeneracy? What is the structure of the ground state subspace, and how do these states relate to the PEPS under consideration?

\subsection{Content of the paper}

In this paper, we introduce a framework for characterizing both MPS and PEPS in terms of symmetries of the underlying tensors. This classification allows us to rederive all results known for one-dimensional systems, while it can be equally applied to the characterization of two- and higher-dimensional PEPS states, answering the aforementioned questions: Using the characterization based on symmetries, we prove how PEPS appear as ground states of local Hamiltonians with finitely degenerate ground states, and how these states can be obtained as variants of the original state. Subsequently, we demonstrate the power of our framework by using it to explain in a simple and coherent way the topological properties of these ground states: We prove that these states are locally indistinguishable and can be transformed into each other by acting on a restricted region, we explain the origin of the topological entropy, and we discuss how to renormalize these states based on their symmetries. We also discuss the excitations of these Hamiltonians, and demonstrate how to understand their anyonic statistics as a consequence of their symmetries. Thus, the characterization of PEPS in terms of symmetries provides a powerful framework which allows to explain a large range of their properties in a coherent and natural way.

The material in this paper is structured as follows: In Sec. 2, we introduce MPS and PEPS and discuss their basic properties. In Sec. 3, we review the proof for the "injective" case, in which the MPS appears as a unique ground state of the parent Hamiltonian. In Sec. 4, we show how general MPS can be classified in terms of symmetries, and use this description to generalize the results of the preceding section; we also discuss how our results relate to the ones obtained using the original canonical form of [13. In Sec. 5. we generalize the symmetry-based classification of MPS to the case of PEPS, where we derive the 
parent Hamiltonian and characterize the structure of its ground state space. In Sec. 6. we consider a more restricted class of PEPS with symmetries, for which we derive a variety of results concerning the structure of the ground state space and the parent Hamiltonian, such as topological entropy, local indistinguishability, renormalization transformations, or the fact that the parent Hamiltonian commutes. We conclude the section by characterizing the excitations of the system and explaining how their anyonic statistics emerges from the symmetries of the PEPS. Finally, in Sec. 7, we discuss examples which illustrates the applicability of our classification, before we conclude in Sec. 8 .

\section{MPS and PEPS}

We start by defining Matrix Product States, which describe the state of a onedimensional chain of $d$-level systems of length $L$.

Definition 2.1. A state $|\psi\rangle \in\left(\mathbb{C}^{d}\right)^{\otimes L}$ is called a Matrix Product State (MPS) if it can be written as

$$
|\mathcal{M}(A)\rangle=\sum_{i_{1}, \ldots, i_{L}} \operatorname{tr}\left[A^{i_{1}} \cdots A^{i_{L}}\right]\left|i_{1}, \ldots, i_{L}\right\rangle .
$$

Here, $A^{i} \in \boldsymbol{L}\left(\mathbb{C}^{D}\right)$ (the space of $D \times D$ matrices over $\mathbb{C}$ ), and $D$ is called the bond dimension.

Note that we restrict to the translational invariant setting with only one tensor $A$, whereas a general MPS can be defined with a different tensor at each site. However, for each MPS describing a translationally invariant state a translationally invariant description (2.1) can be constructed; note, however, that the bond dimension can increase with $L$.

In a more graphical representation, $A$ can be written as a three-index tensor,

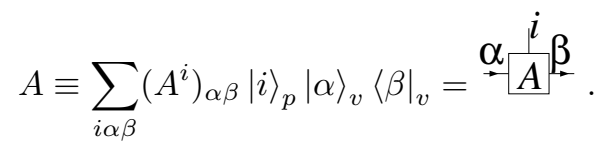

Here, $p$ refers to the "physical" index [characterizing the actual state in (2.1)], and $v$ to the "virtual" index, which is only used to construct the MPS 2.1) and does not appear in the final state. For clarity, we assign arrows to the virtual indices, pointing from bras $\langle\cdot|$ to kets $|\cdot\rangle$. Connecting the legs of two tensors (called a "bond") denotes contraction,

$$
\sum_{\beta}\left(A^{i}\right)_{\alpha \beta}\left(A^{j}\right)_{\beta \gamma}|i, j\rangle_{p}|\alpha\rangle_{v}\left\langle\left.\gamma\right|_{v}=\stackrel{\alpha}{\stackrel{i}{A} \beta} \stackrel{j}{A}{ }^{j} .\right.
$$

Thus, we can write the MPS 2.1] as

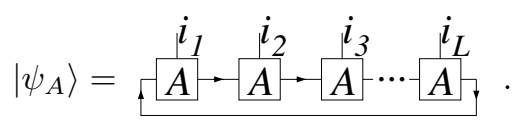


Having this picture in mind, we can immediately define a two-dimensional generalization:

Definition 2.2. A state $|\psi\rangle \in\left(\mathbb{C}^{d}\right)^{\otimes(L \times L)}$ is called a Projected Entangled Pairs State (PEPS) of bond dimension $D$ if it can be written as

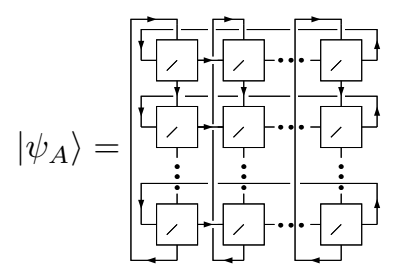

with $A_{\alpha \beta \gamma \delta}^{i}, i=1, \ldots, d, \alpha, \beta, \gamma, \delta=1, \ldots, D$ a five-index tensor, where the index denoted $i$ corresponds to the physical system and "goes out of the paper".

Note that in the definition 2.2 we have implicitly defined the direction of the arrows, and thus the assignments of kets and bras in $A$ :

$$
A \equiv \sum_{i l t r b} A_{l t r b}^{i}|i\rangle_{p}|l, t\rangle_{v}\langle r,\left.b\right|_{v} \equiv l \rightarrow \underbrace{i_{b} t}_{v_{b}} \rightarrow r .
$$

Let us now introduce two simplifications to the MPS tensors, which will allow us to characterize all MPS based on their non-local properties.

First, we define the "projector" - the map which creates the physical system from the virtual layer.

Definition 2.3. For an MPS given by a tensor $A$,

$$
\mathcal{P}(A):=\sum_{i \alpha \beta} A_{\alpha \beta}^{i}|i\rangle_{p}\left\langle\alpha,\left.\beta\right|_{v},\right.
$$

is the map which maps the virtual system to the physical one. The definition extends directly to PEPS.

The importance of the map $\mathcal{P}(A)$ lies in the fact that it tells us how virtual and the physical space of the MPS are connected. On the one hand, it tells us which physical states can be created by acting on the virtual degrees of freedom, and on the other hand, it tells us which virtual configurations - which in turn enforce physical configurations on the surrounding sites - can be realized by acting on the physical system. Thus, studying the properties of $\mathcal{P}(A)$ will allow us to infer properties of the MPS.

Let us now see which simplifications we can make in the analysis of $\mathcal{P}(A)$, given that we are only interested in the non-local properties of the MPS (PEPS). Define $\mathcal{R}(A):=\operatorname{rg} \mathcal{P}(A)$, and $\mathcal{D}(A):=(\operatorname{ker} \mathcal{P}(A))^{\perp}=\operatorname{span}\left(\left\{A^{i}\right\}_{i}\right)$. Then, $\mathcal{P}(A)$ can be inverted on $\mathcal{D}(A)$ and $\mathcal{R}(A)$, respectively:

$$
\exists \mathcal{P}(A)^{-1}: \mathcal{P}(A)^{-1} \mathcal{P}(A)=\left.\mathbb{1}\right|_{\mathcal{D}(A)} \wedge \mathcal{P}(A) \mathcal{P}(A)^{-1}=\left.\mathbb{1}\right|_{\mathcal{R}(A)} .
$$


Note that $\mathcal{R}(A)$ characterizes the local support of the MPS: Projecting sites $2, \ldots, L$ on any basis state $i_{2}, \ldots, i_{L}$ leaves us with $\sum_{i_{1}} \operatorname{tr}\left[A^{i_{1}} X\right]\left|i_{1}\right\rangle$ (where $\left.X=A^{i_{2}} \cdots A^{i_{L}}\right)$, and thus, the single-site reduced operator is supported on $\mathcal{R}(A)$. Thus, we can restrict the MPS to the subspace $\mathcal{R}(A)^{\otimes L}$, i.e., each local system can be mapped to a $\operatorname{dim} \mathcal{R}(A)$-dimensional system by a local isometry, i.e., without changing any non-local properties. This implies that we can w.l.o.g. restrict our analysis to MPS with the following property.

Observation 2.4. Any MPS/PEPS $|\mathcal{M}(A)\rangle$ can be characterized (up to local isometries) by a tensor $A$ for which $\mathcal{P}(A)$ has a right inverse (denoted by $A^{-1}$ in the diagram) such that:

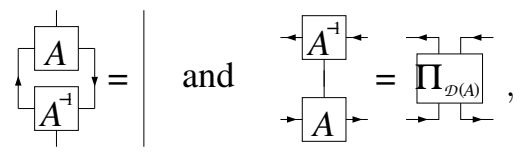

where $\left.\Pi_{\mathcal{D}(A)} \equiv \mathbb{1}\right|_{\mathcal{D}(A)}$ is the orthogonal projector on $D(A)$. W.l.o.g., we will assume a description with this property from now on.

The purpose of this paper is to characterize MPS and PEPS by looking at the structure of the subspace $\mathcal{D}(A)$, and especially at its symmetries. As it turns out, it is sufficient to consider two cases: First, $\mathcal{D}(A)=\operatorname{span}\left(\left\{A^{i}\right\}_{i}\right)=$ $\boldsymbol{L}\left(\mathbb{C}^{D}\right)$, the space of all linear operators on $\mathbb{C}^{D}$, and second, the case where $\mathcal{D}(A)=\operatorname{span}\left(\left\{A^{i}\right\}_{i}\right)$ is an arbitrary $\mathrm{C}^{*}$-algebra, i.e., a linear space of matrices closed under multiplication and hermitian conjugation.

In order to see why we only need to consider these two cases, take an MPS with tensor $A$, and group its sites into super-blocks of $k$ sites each. This results in a new MPS with tensor

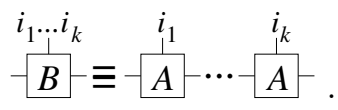

The map $\mathcal{P}(B)$ for this new tensor goes from a $D^{2}$ to a $d^{k}$-dimensional space: Thus, for $k>2 \log _{d} D$, we have that $D^{2}<d^{k}$, which means that the map $\mathcal{P}(B)$ will typically be injective, and thus $D(A)=\boldsymbol{L}\left(\mathbb{C}^{D}\right) 1$ These MPS are known as injective, and they appear as unique ground states of their associated parent Hamiltonians.

The second case $-D(A)$ being a $\mathrm{C}^{*}$-algebra - arises e.g. if the $A^{i}$ are block diagonal matrices, but within each block span the whole space of linear operators. As shown in 13, this does in fact cover the case of a general MPS, as any MPS can be brought into this form. While these states are no longer unique ground states of local Hamiltonians, their parent Hamiltonians have a finite ground state degeneracy, and the ground states are described by the individual blocks of the $A_{i}$.

In the following, we will first review the situation of injective MPS, and show how to prove that they are unique ground states of local Hamiltonians.

\footnotetext{
${ }^{1}$ See 13 for a discussion of how to understand "typical" in this context.
} 
We will then turn towards the case where $\mathcal{D}(A)$ is a general $\mathrm{C}^{*}$-algebra, which we translate into a condition on the symmetry of $A$ under unitaries. While in one dimension this reproduces the results previously derived using the block structure of the matrices $A^{i}$ [11, 13, it will enable us to generalize these results to the two-dimensional scenario, where we will find states exhibiting topological order and anyonic excitations, all of which can be understand purely in terms of symmetries.

\section{MPS: the injective case}

\subsection{Definition and basic properties}

We start by analyzing the injective case in which $D(A)=\operatorname{span}\left(\left\{A^{i}\right\}_{i}\right)=\boldsymbol{L}\left(\mathbb{C}^{D}\right)$. According to Observation 2.4 we can choose $A$ such that $\mathcal{P}(A)$ is invertible. This leads us to the following formal definition of injective.

Definition 3.1 (Injectivity). A tensor $A$ is called injective if $\mathcal{P}(A)$ has a left inverse

$$
\left.\begin{array}{l}
-A^{-1} \\
-A
\end{array}=\right][\text {. }
$$

(The corresponding $M P S|\mathcal{M}(A)\rangle$ will also be termed injective.) Intuitively, injectivity means that we can achieve any action on the virtual indices by acting on the physical spins.

Lemma 3.2 (Stability under concatenation). The injectivity property (3.1) is stable under concatenation of tensors: If $A$ and $B$ are injective, then the tensor obtained by concatenating $A$ and $B$ is also injective, since

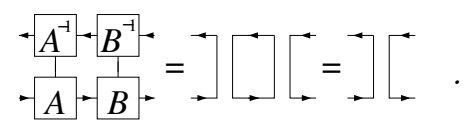

Note that we generally omit normalization constants in the diagrams (the contraction of the loop is $\operatorname{tr} \mathbb{1}=D$ ).

\subsection{Parent Hamiltonians}

Let us now see how injective MPS give rise to parent Hamiltonians, to which they are unique ground states. To this end, note that the two-particle reduced operator of the MPS $|\mathcal{M}(A)\rangle$ is given by

$$
\rho^{[2]}(A)=\sum_{i_{3}, \ldots, i_{L}} \psi_{i_{3}, \ldots, i_{L}}^{[2]}(A)
$$

with $\psi_{i_{3}, \ldots, i_{L}}^{[2]}(A)$ the projector onto the two-particle state obtained by projecting sites $3, \ldots, L$ to the basis state $i_{3}, \ldots, i_{L}$,

$$
\left|\psi_{i_{3}, \ldots, i_{L}}^{[2]}(A)\right\rangle=\sum_{i_{1}, i_{2}} \operatorname{tr}\left[A^{i_{1}} A^{i_{2}} X^{i_{3}, \ldots, i_{L}}\right]\left|i_{1}, i_{2}\right\rangle
$$


with $X^{i_{3}, \ldots, i_{L}}=A^{i_{3}} \cdots A^{i_{L}} \in D(A)$. Thus, $\rho^{[2]}(A)$ is supported on the subspace

$$
\mathcal{S}_{2}=\left\{\sum_{i} \operatorname{tr}\left[A^{i} A^{j} X\right]|i, j\rangle \mid X \in \boldsymbol{L}\left(\mathbb{C}^{D}\right)\right\}=\left\{\begin{array}{cc}
i^{i} \\
A \\
-X
\end{array} \mid X \in \boldsymbol{L}\left(\mathbb{C}^{D}\right)\right\} .
$$

Moreover, the injectivity of $A$ implies that $X^{i_{3}, \ldots, i_{L}}$ spans the space of all $D \times D$ matrices, and thus, $\rho^{[2]}$ has actually full rank on $\mathcal{S}_{2}$. Analogously to 3.2$]$, one can define a sequence of subspaces

$$
\mathcal{S}_{k}=\left\{\sum_{i_{1}, \ldots, i_{k}} \operatorname{tr}\left[A^{i_{1}} \cdots A^{i_{k}} X\right]\left|i_{1}, \ldots, i_{k}\right\rangle \mid X \in \boldsymbol{L}\left(\mathbb{C}^{D}\right)\right\}
$$

which by the same arguments exactly support the $k$-body reduced operator $\rho^{[k]}(A)$.

The idea for obtaining a parent Hamiltonian is now as follows: Define a twobody Hamiltonian which has $\mathcal{S}_{2}$ as its ground state subspace, and let the parent Hamiltonian be the sum of these local terms. The proof consists of two parts: First, we show that for a chain of length $k$ with open boundaries, the ground state subspace is $\mathcal{S}_{k}$ (i.e., optimal), and second, when closing the boundaries, the only state remaining is $|\mathcal{M}(A)\rangle$. This is formalized in the following two theorems.

Theorem 3.3 (Intersection property). Let $A$ and $B$ be injective tensors. Then,

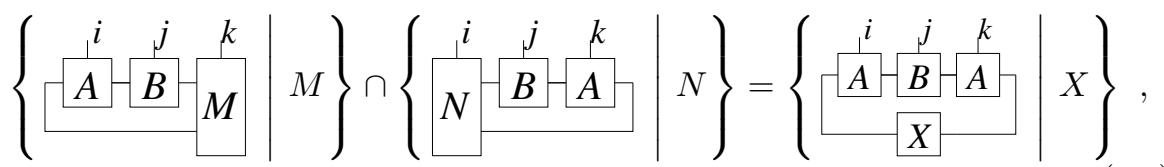

with $M, N, X \in \boldsymbol{L}\left(\mathbb{C}^{D}\right)$.

Proof. It is clear that the right side is contained in the intersection, since for any $X$ we can choose

$$
-M=\frac{-1}{-X} \text { and } N=\frac{1}{-X} .
$$

Conversely, for any $|\psi\rangle$ in the l.h.s. of $(3.3)$, there exist $M, N$ such that

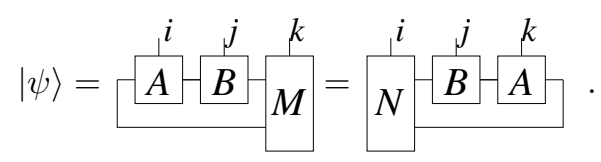

Applying the left inverse of $\mathcal{P}(A)$ and $\mathcal{P}(B)$ to the $i$ and $j$ index, we find that

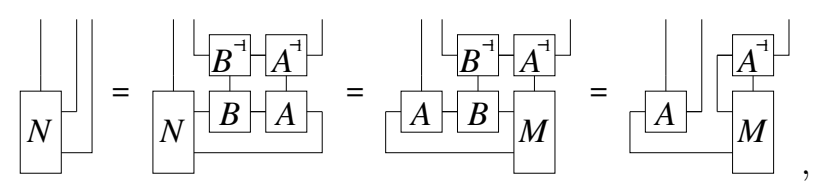

i.e., $N$ is of the form (3.4), and thus $|\psi\rangle$ is contained in the r.h.s. of 3.3. 
Theorem 3.4 (Closure property). For injective $A$ and $B$,

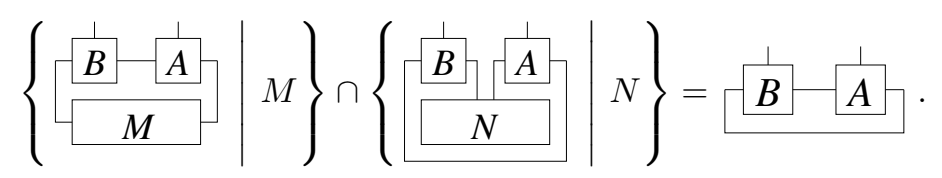

Proof. As in the proof of Theorem 3.3 , the r.h.s. is trivially contained in the intersection by choosing $N=M=\mathbb{1}$. Conversely, by taking an arbitrary element in the intersection and applying the left inverses of $\mathcal{P}(A)$ and $\mathcal{P}(B)$, we find that

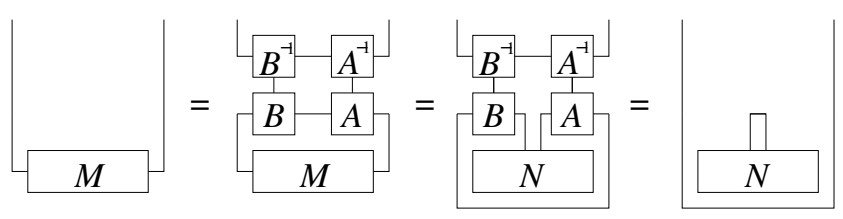

which proves (3.5).

Let us now put Theorems 3.3 and 3.4 together to show that the MPS $|\mathcal{M}(A)\rangle$ arises as the unique frustration free ground state of a local Hamiltonian.

Theorem 3.5 (Parent Hamiltonians). Let $A$ be injective, and $\mathcal{S}_{2}$ as in Eq. (3.2). Define

$$
h_{i}=\mathbb{1}_{d}^{\otimes(i-1)} \otimes\left(1-\Pi_{\mathcal{S}_{2}}\right) \otimes \mathbb{1}_{d}^{L-i-2}
$$

as the orthogonal projector on the subspace orthogonal to $\mathcal{S}_{2}$ on sites $i$ and $i+1$ (modulo L). Then,

$$
H_{\mathrm{par}}=\sum_{i=1}^{L} h_{i}
$$

has $|\mathcal{M}(A)\rangle$ as its unique and frustration free ground state.

Proof. For $k<L$, define $\boldsymbol{j}[k-1]:=\left(i_{2}, \ldots, i_{k-1}\right)$ and $B^{j[k-1]}:=A^{i_{2}} \cdots A^{i_{k-1}}$. Rewriting

$$
\mathcal{S}_{k-1} \otimes \mathbb{C}^{d}=\left\{\sum_{i_{1}, \boldsymbol{j}[k-1], i_{k}} \operatorname{tr}\left[A^{i_{1}} B^{\boldsymbol{j}[k-1]} M^{i_{k}}\right]\left|i_{1}, \boldsymbol{j}[k-1], i_{k}\right\rangle \mid M \in \mathbb{C}^{d} \otimes \boldsymbol{L}\left(\mathbb{C}^{D}\right)\right\}
$$

(and similarly for $\mathbb{C}^{d} \otimes \mathcal{S}_{k-1}$ and $S_{k}$ ), Theorem 3.3 implies that $\mathcal{S}_{k-1} \otimes \mathbb{C}^{d} \cap$ $\mathbb{C}^{d} \otimes \mathcal{S}_{k-1}=\mathcal{S}_{k}$, and thus by induction

$$
\mathcal{S}_{L}=\mathcal{S}_{2} \otimes\left(\mathbb{C}^{d}\right)^{\otimes(k-2)} \cap \mathbb{C}^{d} \otimes \mathcal{S}_{2} \otimes\left(\mathbb{C}^{d}\right)^{\otimes(k-3)} \cap \cdots \cap\left(\mathbb{C}^{d}\right)^{\otimes(k-2)} \otimes \mathcal{S}_{2}, \text { (3.6) }
$$

i.e., the subspace supporting the length $L$ chain is given by the intersection of the two-body supports $\mathcal{S}_{2}$. 
Now let $H_{\text {left }}=h_{1}+\frac{1}{2} h_{2}+\cdots+\frac{1}{2} h_{L-1}$, and $H_{\text {right }}=\frac{1}{2} h_{2}+\cdots+\frac{1}{2} h_{L-1}+h_{L}$. As the $h_{i}$ are projectors, the null space of $H_{\text {left }}$ is given by the intersection (3.6), i.e., by

$$
\mathcal{S}_{\text {left }}=\mathcal{S}_{L}=\left\{\sum_{i_{1}, \boldsymbol{j}[k]} \operatorname{tr}\left[A^{i_{1}} B^{j[k]} M\right]\left|i_{1}, \boldsymbol{j}[k]\right\rangle \mid M \in \boldsymbol{L}\left(\mathbb{C}^{D}\right)\right\}
$$

Correspondingly, the null space of $H_{\text {right }}$ is

$$
\mathcal{S}_{\text {right }}=\left\{\sum_{i_{1}, \boldsymbol{j}[k]} \operatorname{tr}\left[A^{i_{1}} N B^{j[k]}\right]\left|i_{1}, \boldsymbol{j}[k]\right\rangle \mid N \in \boldsymbol{L}\left(\mathbb{C}^{D}\right)\right\},
$$

and thus by the closure property, Theorem 3.4. $|\mathcal{M}(A)\rangle=\mathcal{S}_{\text {left }} \cap \mathcal{S}_{\text {right }}$ is the unique zero-energy (i.e., frustration free) ground state of $H_{\text {par }}=H_{\text {left }}+H_{\text {right }}$.

Note that all these results hold equally for injective PEPS [51]. We will discuss the case of PEPS in detail for the non-injective scenario, which includes the injective one as a special case. Note also that the results of this section do not rely on the translational invariance of the PEPS - the central Theorems 3.3 and 3.4 hold for any pair $A, B$ of injective tensors.

\section{MPS: the $G$-injective case}

\subsection{Definition and basic properties}

In the following, we will consider the case where $A$ is not injective, but where nevertheless

$$
\mathcal{D} \equiv \mathcal{D}(A)=\left\{\sum_{i} \lambda_{i} A^{i} \mid \lambda_{i} \in \mathbb{C}\right\}
$$

forms a $\mathrm{C}^{*}$-algebra.

In the following, we will characterize the structure of $\mathcal{D}$, and thus of $A$, in terms of symmetries. Using Observation 2.4 - that $\mathcal{P}(A)$ has a left inverse on $\mathcal{D}$ - together with an appropriate characterization of $\mathcal{D}$ will allow us to base proofs on this left inverse, similar to the injective case.

Theorem 4.1. For any $\mathrm{C}^{*}$-algebra $\mathcal{D} \subset \boldsymbol{L}\left(\mathbb{C}^{D}\right)$ there exists a finite group $G$ and a unitary representation $g \mapsto U_{g}$ such that $\mathcal{D}$ is the commutant of $U_{g}$, i.e.,

$$
\mathcal{D}=\left\{X \in \boldsymbol{L}\left(\mathbb{C}^{D}\right) \mid\left[X, U_{g}\right]=0 \forall g \in G\right\} .
$$

Proof. Any $\mathrm{C}^{*}$-algebra $\mathcal{D}$ can be decomposed as

$$
\mathcal{D} \cong \bigoplus_{i=1}^{I} \mathbb{1}_{d_{i}} \otimes \boldsymbol{L}\left(\mathbb{C}^{m_{i}}\right),
$$


where $\cong$ denotes unitary equivalence, $A \cong B: \Leftrightarrow \exists W, W W^{\dagger}=\mathbb{1}: A=W B W^{\dagger}$. Now choose a finite group $G$ and a representation $U_{g}$ with irreducible representations $D^{i}$ of dimensions $d_{i}$ and multiplicity $m_{i}$, respectively ${ }_{2}^{2}$

$$
U_{g} \cong \bigoplus_{i=1}^{I} D^{i}(g) \otimes \mathbb{1}_{m_{i}} .
$$

Now Schur's lemma implies Eq. 4.2.

Note that conversely, any unitary representation $U_{g}$ has a $\mathrm{C}^{*}$-algebra as its commutant (4.2), since $U_{g}^{\dagger}=U_{g^{-1}}$. Thus, the characterization in terms of unitaries is equivalent to the characterization in terms of $\mathcal{D}$, the span of the $A^{i}$. This motivates the following definition.

Definition 4.2. Let $g \mapsto U_{g}$ be a unitary representation of a finite group $G$. We say that an MPS tensor $A$ is $G$-injective if

i) $\forall i, g: U_{g} A^{i} U_{g}^{\dagger}=A^{i}$, and

ii) the map $\mathcal{P}(A)[E q$. 2.3] has a left inverse on the subspace

$$
\mathcal{S}=\left\{X \mid\left[X, U_{g}\right]=0\right\}
$$

of $U_{g}$-invariant matrices,

$$
\mathcal{P}^{-1}(A) \mathcal{P}(A)=\left.\mathbb{1}\right|_{\mathcal{S}} .
$$

In graphical notation, we will denote unitary representations as circles labelled $U_{g}$, or simply $g$ when unambigous. Note that the arrow now point towards the ket on which to apply $U_{g}$. Condition i) of Definition 4.2 then reads

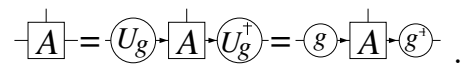

Note that the group $G$ is more important than its representation $U_{g}$ (though we will require certain properties at some point), and in fact, a different representation can be attached to each bond.

Lemma 4.3. The orthogonal projector $\boldsymbol{\sigma}$ onto the $U_{g}$-invariant subspace 4.4 is given by

$$
\boldsymbol{\sigma}(X)=\frac{1}{|G|} \sum_{g} U_{g} X U_{g}^{\dagger} .
$$

Here, $|G|$ is the cardinality of the group $G$. Thus, condition 4.5 corresponds to

$$
\begin{aligned}
& \sqrt{A^{-1}} \\
& =A
\end{aligned}=\sum_{g} \vec{g} \cdot \overrightarrow{g^{+}} .
$$

(We generally omit normalization in diagrams.)

\footnotetext{
2 This can be achieved, e.g., by choosing $I$ finite groups $G_{i}$ which have a $d_{i}$-dimensional irreducible representation $\tilde{D}_{i}$, and letting $G=G_{1} \times \cdots \times G_{I}$, and $D^{i}\left(g_{1}, \ldots, g_{I}\right)=\tilde{D}_{i}\left(g_{i}\right)$. In particular, the choice of $G$ and $U_{g}$ is not unique.
} 
Proof. Since

$$
\begin{aligned}
\boldsymbol{\sigma}^{2}(X) & =\frac{1}{|G|^{2}} \sum_{g h} U_{h} U_{g} X U_{g}^{\dagger} U_{h}^{\dagger} \\
& =\frac{1}{|G|^{2}} \sum_{h g} U_{h g} X U_{h g}^{\dagger}=\frac{1}{|G|} \sum_{k} U_{k} X U_{k}^{\dagger}=\boldsymbol{\sigma}(X),
\end{aligned}
$$

$\boldsymbol{\sigma}$ is a projection. As it leaves $\mathcal{S}$ invariant,

$$
U_{h} \boldsymbol{\sigma}(X) U_{h}^{\dagger}=\frac{1}{|G|} \sum_{g} U_{h g} X U_{h g}^{\dagger}=\boldsymbol{\sigma}(X)
$$

(i.e., the image is contained in $\mathcal{S}$ ), and it is hermitian, it is the orthogonal projector on $\mathcal{S}$.

We will now show the analogue of Lemma 3.2 G-injectivity is stable under concatenation of tensors. To this end, we will use the following identity.

Lemma 4.4. For any unitary representation $U_{g}$ of a finite group,

$$
\sum_{h} g^{\Delta} \frac{\vec{a}}{h}=5
$$

with

$$
\Delta \cong \frac{1}{|G|} \bigoplus_{i} \frac{d_{i}}{m_{i}} \mathbb{1}_{d_{i}} \otimes \mathbb{1}_{m_{i}},
$$

where $\left(d_{i}, m_{i}\right)$ are dimensions and multiplicites of the irreducible representations $D^{i}$ of $U_{g}$, and $\Delta$ is diagonal in the basis in which $U_{g} \cong \bigoplus_{i} D^{i}(g) \otimes \mathbb{1}_{m_{i}}$. In a formula,

$$
\sum_{h} \operatorname{tr}\left[U_{h}^{\dagger} \Delta U_{g}\right] U_{h}=\sum_{h} \operatorname{tr}\left[U_{g h^{-1}} \Delta\right] U_{h}=U_{g} .
$$

Proof. The group orthogonality theorem implies

$$
\begin{aligned}
\sum_{g} \operatorname{tr}\left[D^{i}\left(g^{-1}\right)\right] D^{j}(g) & =\sum_{g, m, k, l} \bar{D}_{m m}^{i}(g) D_{k l}^{j}(g)|k\rangle\langle l| \\
& =\frac{|G|}{d_{i}} \sum_{m, k, l} \delta_{i, j} \delta_{m, k} \delta_{m, l}|k\rangle\langle l| \\
& =\frac{|G|}{d_{i}} \delta_{i, j} \mathbb{1}_{d_{i}} .
\end{aligned}
$$


Thus,

$$
\begin{aligned}
\sum_{h} \operatorname{tr}\left[U_{g h^{-1}} \Delta\right] U_{h} & =\sum_{k} \operatorname{tr}\left[U_{k^{-1}} \Delta\right] U_{k} U_{g} \\
& \cong \sum_{k}\left[\sum_{i} m_{i} \operatorname{tr}\left[D^{i}\left(k^{-1}\right) \frac{d_{i}}{m_{i}|G|}\right]\right] \bigoplus_{j}\left[D^{j}(k) D^{j}(g)\right] \otimes \mathbb{1}_{m_{i}} \\
& =\bigoplus_{j} D^{j}(g) \otimes \mathbb{1}_{m_{i}} \\
& \cong U_{g} .
\end{aligned}
$$

For a restricted class of representations, we can make a stronger statement.

Definition 4.5 (Semi-regular representations). A unitary representation $g \mapsto$ $U_{g}$ of a finite group $G$ is called semi-regular if $U_{g}$ contains all irreducible representations of $G$.

Lemma 4.6 (Linear independence of semi-regular representations). Let $g \mapsto U_{g}$ be a semi-regular representation of a group $G$. Then,

$$
\operatorname{tr}\left[U_{g}^{\dagger} U_{h} \Delta\right]=\delta_{g, h}
$$

with $\Delta$ as of 4.6. Note that for the regular representation, $\Delta \propto \mathbb{1}$.

Proof. This follows as $|G| \operatorname{tr}\left[U_{k} \Delta\right]=|G| \sum_{i} m_{i} \operatorname{tr}\left[\frac{d_{i}}{m_{i}|G|} D^{i}(k)\right]$ is the character of the regular representation, which is $|G| \delta_{k, 1}$.

We are now ready to prove the analogue of Lemma 3.2 for the $G$-injective case.

Lemma 4.7 (Stability under concatenation). Let $A$ and $B$ be $G$-injective tensors. Then $C^{i j}=A^{i} B^{j}$ is also $G$-injective with left inverse

$$
-C^{-1}-:=-A_{1}^{-1} \cdot \Delta \cdot-\underline{B^{-1}} \cdot .
$$

Here, $\Delta$ is defined as in Lemma 4.4.

Proof. $G$-invariance of $C$ follows from

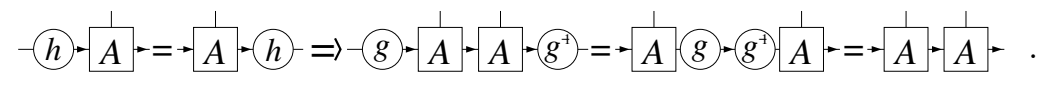

Moreover, 4.9 is the left inverse of $\mathcal{P}(C)$ on the $U_{g}$-invariant subspace since

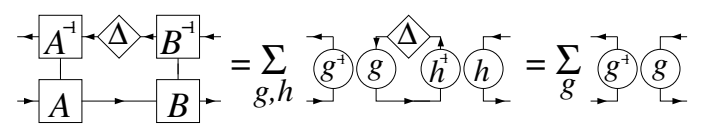

from Lemma 4.4 . 


\subsection{Parent Hamiltonians}

Let us now proceed to the relation of $G$-injective MPS and parent Hamiltonians. The construction is exactly analogous to the injective case: We define

$$
\mathcal{S}_{k}=\left\{\sum_{i_{1}, \ldots, i_{k}} \operatorname{tr}\left[A^{i_{1}} \cdots A^{i_{k}} X\right]\left|i_{1}, \ldots, i_{k}\right\rangle \mid X \in \boldsymbol{L}\left(\mathbb{C}^{D}\right)\right\},
$$

and prove the analogues of Theorem 3.3 (Intersection Property) and Theorem 3.4 (Closure Property), but now for $G$-injective MPS. While the intersection property will be the same as in the injective case, the closure will give rise to a subspace whose dimension equals the number of conjugacy classes of $G$, for a properly chosen group $G$.

Theorem 4.8 (Intersection property). Let $A, B$ be $G$-injective. Then,

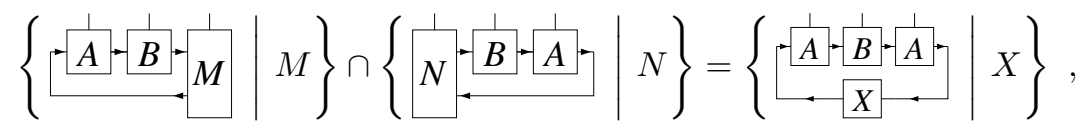

Proof. Using the $G$-invariance of $A$ and $B$, we can infer that we can restrict $M$, $N$, and $X$ to also be $G$ invariant in the virtual indices. For instance, for any $M$,

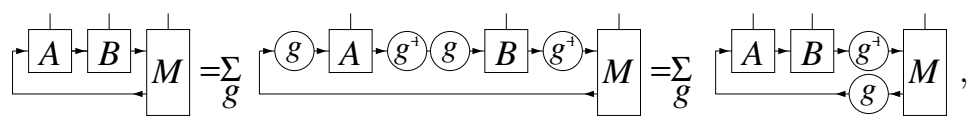

this is, we can replace $M$ by

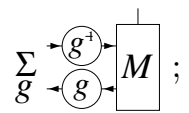

the same holds true for $N$ and $X$. We will always assume symmetrized tensors from now on.

We are now ready to prove (4.11). First, the r.h.s. is clearly contained in the l.h.s., by choosing $M^{i}=A^{i} X, N^{\imath}=X A^{i}$. On the other hand, each element in the intersection can be simultaneously characterized by a pair $N, M$ of tensors, and by applying the inverse maps we find that

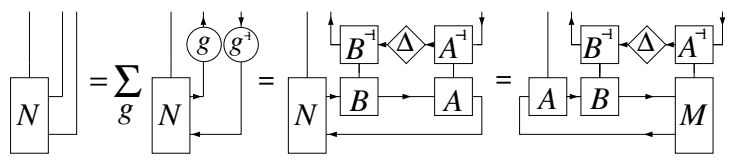

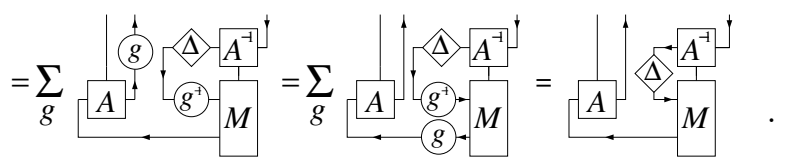

This shows that $M$ (and equally $N$ ) are of the form required by Eq. 4.11, and thus proves the theorem. 
Theorem 4.9 (Closure property). For $G$-injective $A$ and $B$,

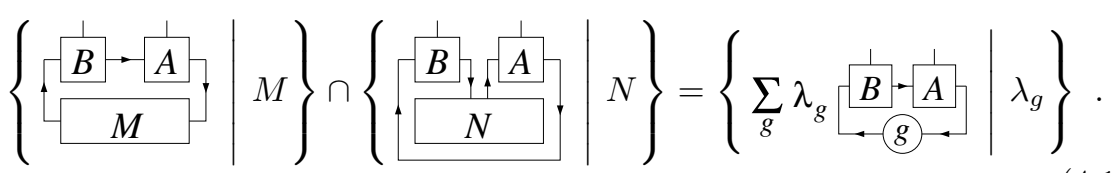

Before proving the theorem, let us give an intuition why the closure can be done using any $U_{g}$ (and nothing else). To this end, regard $B$ as the $(L-1)$-fold blocking of $A$ 's. The closures $U_{g}$ are exactly the operators which commute with $A$, and therefore, they can be moved to any position in the chain. Thus, no local block of $A$ 's needs to hold the closing $U_{g}$, i.e., the state looks the same locally independent of the closure.

Proof. We may again assume that $M$ and $N$ are $G$-invariant. It is clear that the r.h.s. is contained in the l.h.s., by moving $U_{g}$ to the relevant link and setting $M=U_{g}$ or $N=U_{g}$, respectively. On the other hand, any element in the intersection can be written using some $M$ and $N$, for which it holds that

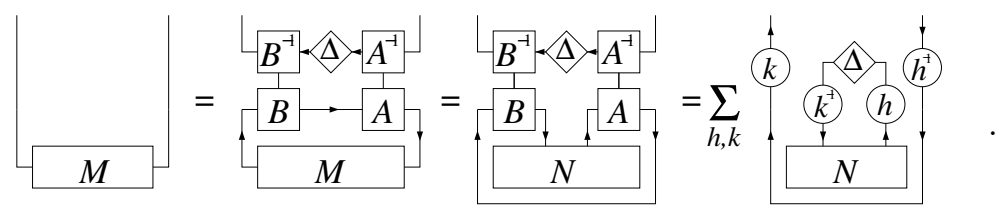

(In the first step, we have used that $M$ is $G$-invariant.) Thus, any element of the intersection is of the form of the r.h.s. of 4.12 , with $\lambda_{g}=\operatorname{tr}\left[U_{g^{-1}} N \Delta\right]$, $g \equiv k h^{-1}$.

Let us now formally define translational invariant MPS with an operator in the closure, as they appear in the above theorem.

Definition 4.10. For an $M P S$ tensor $A$ and $K \in \boldsymbol{L}\left(\mathbb{C}^{D}\right)$, we define

$$
|\mathcal{M}(A \mid K)\rangle:=\sum_{i_{1}, \ldots, i_{L}} \operatorname{tr}\left[A^{i_{1}} \cdots A^{i_{L}} K\right]\left|i_{1}, \ldots, i_{L}\right\rangle
$$

to be the MPS given by $A$ with closure $K^{3}$.

Theorem 4.11 (Parent Hamiltonians). Let $A$ be $G$-injective, $\mathcal{S}_{2}$ as in $(3.2)$, and let

$$
h_{i}=\mathbb{1}_{d}^{\otimes(i-1)} \otimes\left(1-\Pi_{\mathcal{S}_{2}}\right) \otimes \mathbb{1}_{d}^{L-i-2} .
$$

Then,

$$
H_{\mathrm{par}}=\sum_{i=1}^{L} h_{i}
$$

\footnotetext{
${ }^{3}$ Note that one can use a different closure to reduce dramatically the bond dimension. One example is the $\mathrm{W}$-state, whose bond dimension grows to infinity with $\mathrm{L}$ with the standard closure 13 55, but has bond dimension 2 with a different closure.
} 
has a subspace of frustration free ground states spanned by the MPS $\left|\mathcal{M}\left(A \mid U_{g}\right)\right\rangle$ with $U_{g}$-closed boundaries.

Proof. The proof is exactly the same as for Theorem 3.5, except that is is now based on Theorems 4.8 and 4.9 the latter leading to the degeneracy of the ground state subspace.

Theorem 4.12 (Structure of ground state subspace). Let $D^{1}, \ldots, D^{I}$ be the irreducible representations of $U_{g}$, of dimension $d_{i}$. Then, the ground state subspace of Theorem 4.11 is I-fold degenerate, and it is spanned by the MPS $\left|\mathcal{M}\left(A \mid \Pi_{i}\right)\right\rangle$, where

$$
\Pi_{i}=\frac{d_{i}}{|G|} \sum_{g} \operatorname{tr}\left[D^{i}\left(g^{-1}\right)\right] U_{g}
$$

is the projector onto the subspace supporting the irreducible representation $D^{i}$ in $U_{g}$ [proven in 4.8 ] ].

Moreover, if $U_{g}$ is a semi-regular representation, $I$ is equal to the number of conjugacy classes of $G$, and the subspace is spanned by the linearly independent states $\left|\mathcal{M}\left(A \mid U_{g}\right)\right\rangle$, where for each conjugacy class $g$, one representative is chosen.

Note that the first part of the theorem corresponds to the known form of the different ground states, corresponding to the block structure of the $A^{i}$ 's 11,13 , while the second part is the new symmetry-based classification of ground states which can be extended to the two-dimensional scenario.

Proof. First, the $G$-invariance of the $A^{i}$ implies that

$$
\left|\mathcal{M}\left(A \mid U_{g}\right)\right\rangle=\left|\mathcal{M}\left(A \mid U_{h} U_{g} U_{h}^{\dagger}\right)\right\rangle=\left|\mathcal{M}\left(A \mid U_{h g h^{-1}}\right)\right\rangle,
$$

i.e., all closures from the same conjugacy class are equivalent. Let $D^{1}, \ldots, D^{J}$, $J \geq I$, be all irreducible representations of $G$. Since the characters $\chi_{i}(g)=$ $\operatorname{tr}\left[D^{i}(g)\right]$ form an orthonormal set for the space of class functions (i.e. the functions which are constant over conjugacy classes), the ground state space is equally spanned by

$$
\sum_{g} \operatorname{tr}\left[D^{i}\left(g^{-1}\right)\right]\left|\mathcal{M}\left(A \mid U_{g}\right)\right\rangle=\left|\mathcal{M}\left(A \mid \sum_{g} \operatorname{tr}\left[D^{i}\left(g^{-1}\right)\right] U_{g}\right)\right\rangle \propto\left|\mathcal{M}\left(A \mid \Pi_{i}\right)\right\rangle ;
$$

Note that $\Pi_{i}=0$ for $i>I$ (i.e. if $U_{g}$ does not contain $D^{i}$ ).

Conversely, linear independence of $\left|\mathcal{M}\left(A \mid \Pi_{i}\right)\right\rangle, i=1, \ldots, I$ can be seen as follows: For any class function $\lambda_{g}$, and with $C$ the $L$-fold blocking of $A$, we have that

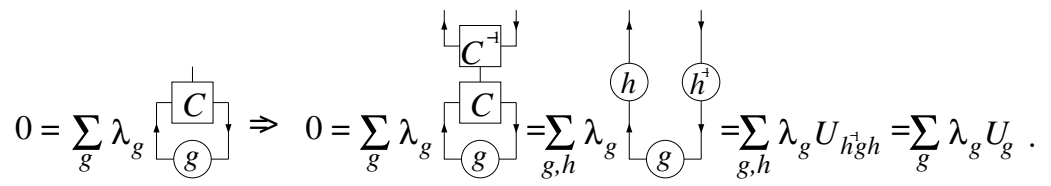


Thus,

$$
\begin{array}{rlrl} 
& 0 & =\sum_{i} \mu_{i}\left|\mathcal{M}\left(A \mid \Pi_{i}\right)\right\rangle=\sum_{i, g} \mu_{i} \frac{d_{i}}{|G|} \operatorname{tr}\left[D^{i}\left(g^{-1}\right)\right]\left|\mathcal{M}\left(A \mid U_{g}\right)\right\rangle \\
\stackrel{4.15}{\longrightarrow} & 0 & =\sum_{i, g} \mu_{i} \frac{d_{i}}{|G|} \operatorname{tr}\left[D^{i}\left(g^{-1}\right)\right] U_{g}=\sum_{i} \mu_{i} \Pi_{i} \\
\Rightarrow \quad 0 & =\mu_{i} \quad \forall i,
\end{array}
$$

which proves linear independence. (Note that we had to use that $D^{i}$ is contained in $U_{g}$, otherwise $\Pi_{i} \equiv 0$.)

The second statement follows from the fact that $\left|\mathcal{M}\left(A \mid U_{g}\right)\right\rangle$ is constant on conjugacy classes, and that the number of conjugacy classes equals the number of irreducible representations of $G$.

\section{Two dimensions: $G$-injective PEPS}

\subsection{Definition and basic properties}

Having understood the one-dimensional case of $G$-injective MPS, let us now turn towards two dimensions. We will introduce some new conventions for the diagrams (as in principle, we need a third dimension), which we will explain right after the definition of $G$-injectivity.

Definition 5.1. Let $g \mapsto U_{g}$ be a semi-regular representation of a finite group $G$. We call a PEPS tensor A $G$-injective if

i) It is invariant under $U_{g}$ on the virtual level,

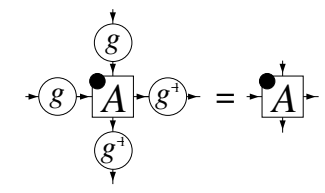

ii) There exists a left inverse to $\mathcal{P}(A)$ such that $\mathcal{P}(A)^{-1} P(A)=\Pi_{\mathcal{U}}$, the projector on the $U_{g}$-invariant subspace:

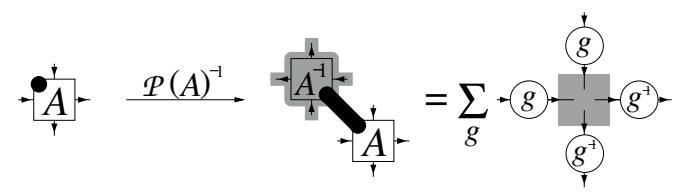

Let us briefly explain the differences in notation: We will try to avoid threedimensional plots as far as possible. PEPS tensors are generally depicted "from the top": The four legs in (5.1) are the virtual indices, and a black dot denotes the physical index (for the inverse, it is in the lower right instead of the upper left corner). As we use the let inverse to make the virtual subspace accessible via the physical indices, we will depict only the situation after applying the left 
inverse whenever possible, as depicted on the very right of (5.2). In order to distinguish the "original" virtual level and the one after the application of the left-inverse, we shade the latter gray. (This corresponds to the lower and upper layer, respectively, in the $1 \mathrm{D}$ case.)

Note that there is no need to choose the same representation $U_{g}$ for the horizontal and vertical direction. In fact, as mentioned earlier representations are assigned to links, and every link can carry its own representation - all that matters is that the two tensors acting on a link act with the same representation. It is this possibility of changing the representations which enables us to prove that $G$-injectivity is is preserved under blocking.

Lemma 5.2 (Stability under concatenation). Let $A$ and $B$ be $G$-injective tensors. Then,

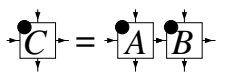

(with blocked up, down, and physical indices) is also G-injective with left inverse

$$
-\frac{1}{A^{-1}}-\Delta-\Delta \cdot-\frac{1}{B^{-1}-}
$$

Proof. First, note that for any two semi-regular representations $U_{g}$ and $V_{g}$, $W_{g}=U_{g} \otimes V_{g}$ is again a semi-regular representation. Then, it is clear that $C$ is also $G$-invariant, as the action of the $U_{g}$ on the inner link cancels. That 5.4 is left-inverse to $C$ follows using $\operatorname{tr}\left[U_{g} \Delta\right] \propto \delta_{g, 1}$, Lemma 4.6

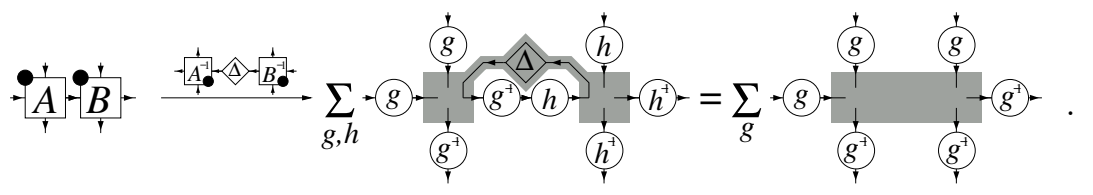

Observation 5.3. Note that $G$-injectivity is also preserved when contracting legs of an already connected block, e.g. the up leg of $A$ with the down leg of $B$ in 5.3): The resulting tensor is clearly again $G$-invariant, and the left-inverse is obtained by contracting the corresponding legs of (5.4) with any any operator with nonzero trace (e.g., $\Delta$ ): The group elements attached to the two legs cancel out, as they belong to the same tensor.

\subsection{Parent Hamiltonians}

The idea to construct parent Hamiltonians is essentially the same as in one dimension. We define the local Hamiltonian as 1 minus the projector on the span of a $2 \times 2$ block (the smallest block which allows for an overlapping tiling of the lattice), and study how the ground state subspace behaves when growing the block. For simplicity, we first grow the $2 \times 2$ block in one direction until we reach a $2 \times L$ lattice, and then in the other direction until we have the full $L \times L$ lattice with open boundaries. Finally, we study what happens when we 
close the boundaries. While the growing will work essentially exactly as in 1D, closing the boundaries will give a richer structure.

Theorem 5.4 (Intersection property). Let A, B be G-injective. Then,

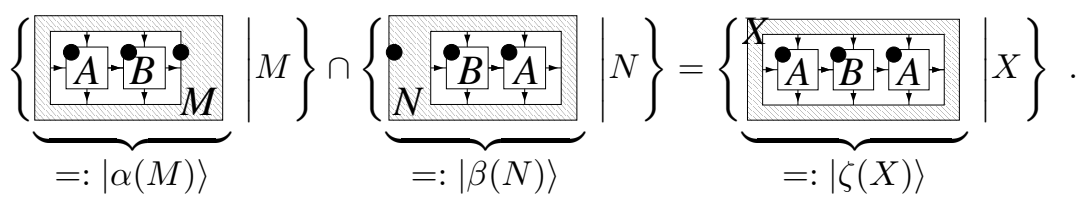

Here, we have chosen to shade the inside of the "boundary condition" tensors $M, N$, and $X$.

Proof. The proof is exactly analogous to the one-dimensional case, Theorem 4.8 The r.h.s. is contained in the l.h.s., as any $|\zeta(X)\rangle$ can be written as both $|\alpha(M)\rangle$ and $|\beta(N)\rangle$. Conversely, any element in the intersection can be written as $|\alpha(M)\rangle=|\beta(N)\rangle$ for some $M$ and $N$; as in the one-dimensional case, we can assume both to be $G$-invariant. To recover $M$, we apply the left inverse (5.4) to the left two physical modes of $|\alpha(M)\rangle=|\beta(N)\rangle$ and obtain

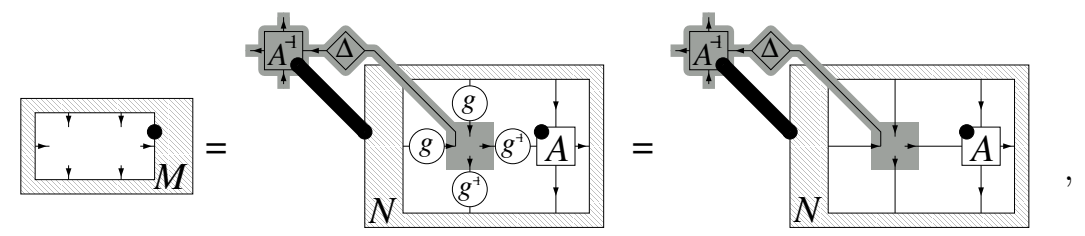

which implies that the state is of the form $|\zeta(X)\rangle$.

Theorem 5.5 (Closure property). For $G$-injective $A, B, C$, and $D$,

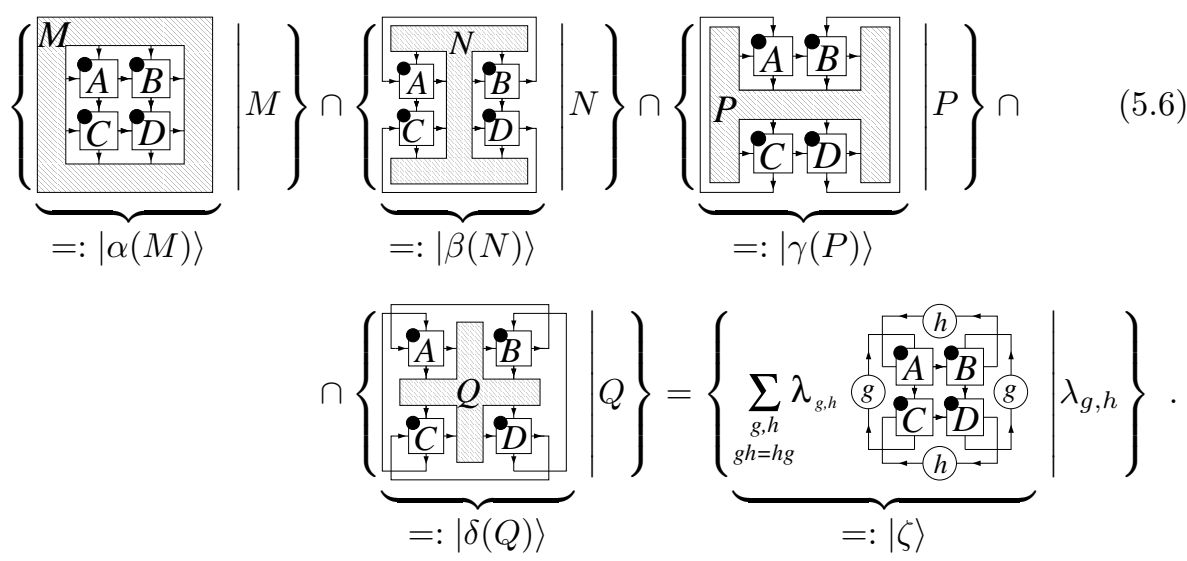

Here, the sum runs over all pairs $(g, h) \in G \times G$ such that $g h=h g$.

Note that if $A, B, C$, and $D$ arise from blocking the original tensor, this corresponds to a closure with two unitaries $U_{g}^{\otimes L}$ and $U_{h}^{\otimes L}$ at the horizontal and vertical closure, respectively. 
Proof. The proof again follows closely the proof for one dimension. First, it is clear that the r.h.s. in contained in the intersection by choosing $M, N, P$, and $Q$ appropriately. To show that every element in the intersection is of the form $|\zeta\rangle$, we consider an element of the intersecion, which can be written as $|\alpha(M)\rangle=|\delta(Q)\rangle$ with boundaries $M$ and $Q$, respectively. As before, we can assume the boundary conditions to be $G$-invariant. To recover $M$, we apply the left-inverse

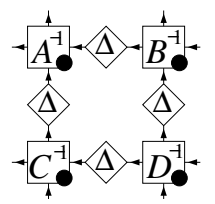

to $|\alpha(M)\rangle=|\delta(Q)\rangle$, and obtain

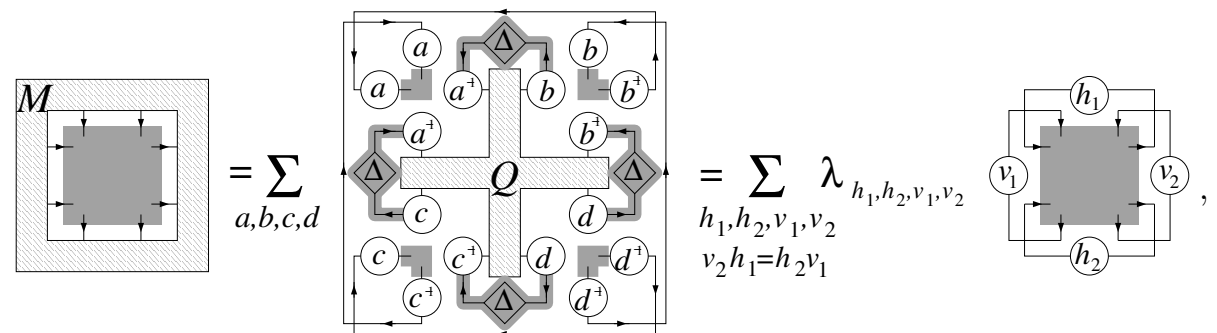

where $h_{1}=b^{-1} a, h_{2}=d^{-1} c, v_{1}=c^{-1} a$, and $v_{2}=d^{-1} b$, which results in the constraint $v_{2} h_{1}=h_{2} v_{1}$ on the sum.

Applying the inverse (5.7) also to $|\alpha(M)\rangle=|\beta(N)\rangle$, we obtain

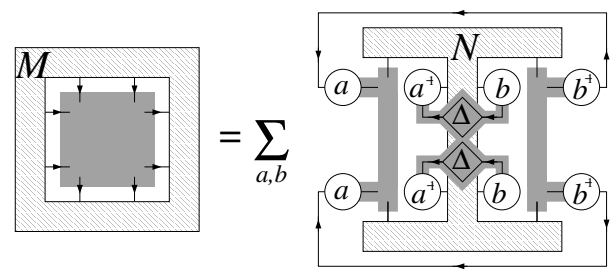

[note that the left and right block of $(5.7)$ are the inverses for the corresponding blocks in $|\beta(N)\rangle]$, which proves that $h_{1}=h_{2}=: h$ in (5.8). Finally, the same for $|\alpha(M)\rangle=|\gamma(P)\rangle$ yields $v_{1}=v_{2}=: g$, and the constraint $v_{2} h_{1}=h_{2} v_{1}$ gives $g h=h g$.

Definition 5.6. For a $G$-injective PEPS tensor $A$, and $g, h \in G$, define

$$
|\mathcal{M}(A \mid(g, h))\rangle:=
$$


to be the $L \times L$ PEPS built from the tensor A, with closures $g$ and $h$. (The representation of $g$ is a property of the bond and omitted for brevity.)

Theorem 5.7 (Parent Hamiltonian). Let $A$ be G-injective,

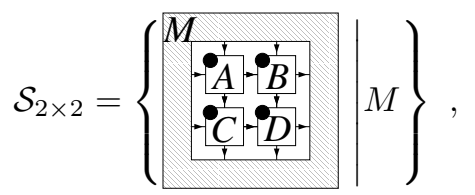

and define

$$
h_{i, j}=\left(1-\Pi_{\mathcal{S}_{2 \times 2}}\right) \otimes \mathbb{1}
$$

where the projector $\Pi_{\mathcal{S}_{2 \times 2}}$ acts on the square $\{i, i+1\} \times\{j, j+1\}$. Then,

$$
H_{\mathrm{par}}=\sum_{i, j=1}^{L} h_{i, j}
$$

has a subspace of frustration free ground states spanned by the PEPS $|\mathcal{M}(A \mid(g, h))\rangle$ with $(g, h)$-closed boundaries, where $g h=h g$.

Proof. The proof goes exactly as in one dimension: We divide the Hamiltonian in four blocks with open boundary conditions (one which does not cover the boundary, two which cover the horizontal or vertical boundary, respectively, and one which covers both boundaries). For each of the blocks, by virtue of the intersection property (Theorem 5.4 the ground state subspace is given by one of the sets in (5.6), and thus, Theorem 5.5 yields the desired result.

Definition 5.8 (Pair-conjugacy classes). For $(g, h) \in G \times G,\left(g^{\prime}, h^{\prime}\right) \in G \times G$, define the equivalence relation

$$
(g, h) \sim\left(g^{\prime}, h^{\prime}\right) \quad: \Leftrightarrow \exists x \in G:(g, h)=\left(x g^{\prime} x^{-1}, x h^{\prime} x^{-1}\right) .
$$

It divides $G \times G$ into disjoint equivalence classes

$$
C[(g, h)]=\left\{\left(g^{\prime}, h^{\prime}\right) \mid(g, h) \sim\left(g^{\prime}, h^{\prime}\right)\right\}
$$

which we call pair-conjugacy classes. To each pair-conjugacy class $C$, we define

$$
|\mathcal{M}(A \mid C)\rangle:=|\mathcal{M}(A \mid(g, h))\rangle \quad(g, h) \in C .
$$

Note that $|\mathcal{M}(A \mid C)\rangle$ is well-defined, since the $g$-invariance of $A$ shows that $|\mathcal{M}(A \mid(g, h))\rangle=\left|\mathcal{M}\left(A \mid\left(g^{\prime}, h^{\prime}\right)\right)\right\rangle$ for $(g, h) \sim\left(g^{\prime}, h^{\prime}\right)$.

Theorem 5.9 (Structure of the ground state subspace). Let $C_{1} \dot{\cup} \cdots \dot{\cup} C_{K} \subset$ $G \times G$ be the pair-conjucacy classes of $G \times G$ for which $g h=h g$ for $(g, h) \in$ $C_{k}$. Then, the ground state subspace of Theorem 5.7 is $K$-fold degenerate and spanned by the states $\left|\mathcal{M}\left(A \mid C_{k}\right)\right\rangle$. 
Proof. The proof resembles the one-dimensional case: As observed in Definition 5.8, closures from the same pair-conjugacy class describe the same state. It remains to show that the states corresponding to different pair-conjugacy classes are linearly independent. Let

$$
0=\sum \lambda_{k}\left|\mathcal{M}\left(A \mid C_{k}\right)\right\rangle=\sum_{k} \lambda_{k}\left|\mathcal{M}\left(A \mid\left(g_{k}, h_{k}\right)\right)\right\rangle,
$$

with $\left(g_{k}, h_{k}\right) \in C_{k}$ representatives of $C_{k}$. Denoting the $L \times L$ block of $A$ 's by $C$, and applying the left inverse of $C$, we have that

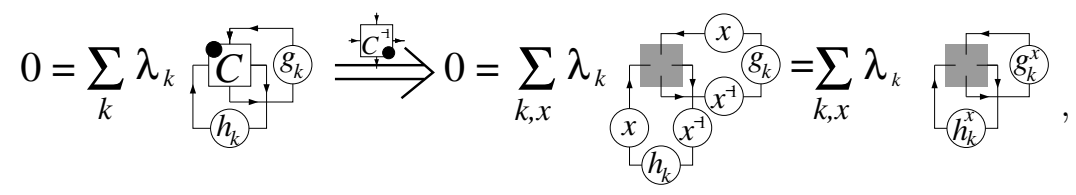

with $\left(g_{k}^{x}, h_{k}^{x}\right) \in C_{k}$ other representatives of $C_{k}$. This is, 5.12 implies that $\sum \lambda_{k} U_{h_{k}^{x}} \otimes U_{g_{k}^{x}}=0$, and since semi-regular representations are linearly independent (Lemma 4.6), this gives $\lambda_{k}=0 \forall k$.

It is straightforward to see that the number of different closures, i.e., the dimension of the ground state subspace, is equal to the number of particle types of the quantum double model of $G[56]$ : A pair-conjugacy class $C \equiv C[(h, k)]$, $h k=k h$, can be characterized by specifying i) the conjugacy class $C[h]$ of $h$, and ii) the conjugacy class of the normalizer $N[h]=\{k: h k=k h\}$ of $h$ which contains $k$. Since the number of conjugacy classes of $N[h]$ is equal to the number of irreducible representations, this is equal to the number of particle types in the $G$ quantum double 57 .

Let us now give an intuitive explanation why the closures should be done using two closed loops of identical unitaries $U_{g}^{\otimes N}$ and $U_{h}^{\otimes N}$, and why one should require $g h=h g$. The key observation here (which will be essential for the introduction of topological excitations later on) is that strings of $U_{g}$ 's can be deformed freely due to the $G$-invariance of $A$. To this end, consider a string of $U_{g}$ 's in the lattice,

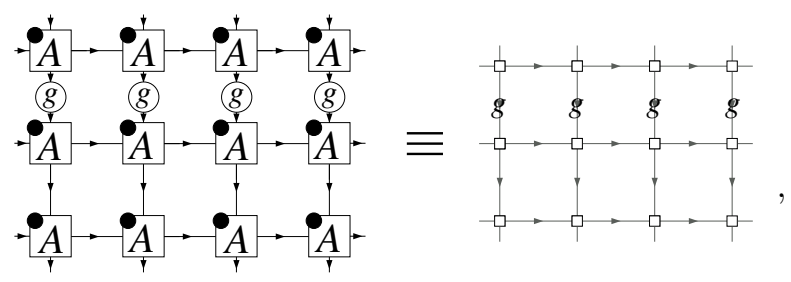

where on the right, we have introduced a new simplified notation which allows for more compact diagrams in situations where we care only about the pattern of $U_{g}$ 's on the virtual level. Using the $G$-invariance of the tensors, we can deform 
the string continuously: e.g.,

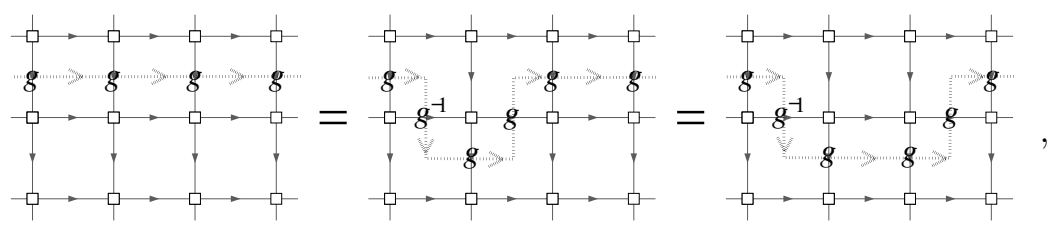

and so further. Note that whether $g$ or $g^{-1}$ has to be used depends on whether the (oriented) string of $U_{g}$ 's crosses the (oriented) edges from the right or from the left.

The possibility to arbitrarily deform strings of $g$ 's and $h$ 's, together with the fact that $g$ and $h$ commute, i.e., the that the two strings don't interact, implies that we can move the loops anywhere we want on the torus. Thus, the closure cannot be detected locally, and the $|\mathcal{M}(A \mid(g, h))\rangle$ are all ground states of the Hamiltonian (5.11). (Note, however, that this does not imply that the reduced density operators are the same, but only that they all live within the same subspace $\mathcal{S}_{2}$. Indeed, in one dimension the reduced operators can look different; e.g., the GHZ state MPS $A^{i}=|i\rangle\langle i|, i=0,1$ gives a Hamiltonian for which all $\alpha|0, \ldots, 0\rangle+\beta|1, \ldots, 1\rangle$ are ground states.

\section{Isometric PEPS}

\subsection{Definition an basic properties}

We will now consider a subclass of $G$-injective PEPS, namely those for which $\mathcal{P}(A)$ is an isometry (mapping the space of $G$-invariant tensors unitarily to the range of $\mathcal{P}(A))$. Using Observation 2.4 which says that we can w.l.o.g. restrict the physical system to the range of $\mathcal{P}(A)$, we obtain that a $\mathcal{P}(A)$ is isometric if $\mathcal{P}(A)^{-1}=\mathcal{P}\left(A^{\dagger}\right)$, where the dagger is with respect to the virtual levels:

$$
A=\sum_{i \alpha \beta \gamma \delta} A_{\alpha \beta \gamma \delta}^{i}|i\rangle_{p}|\alpha, \beta\rangle\left\langle\gamma, \delta\left|\rightarrow A^{\dagger}:=\sum_{i \alpha \beta \gamma \delta} \bar{A}_{\alpha \beta \gamma \delta}^{i}\right| i\right\rangle_{p}|\gamma, \delta\rangle\langle\alpha, \beta| .
$$

(Loosely speaking, the reason to use the dagger instead of the conjugation is that the edges for $\mathcal{P}(A)$ and $\mathcal{P}(A)^{-1}$ have opposite orientation.)

For clarity, we will illustrate proofs for the one-dimensional case where appropriate.

Definition 6.1. A G-injective PEPS is called $G$-isometric if $U_{g} \equiv L_{g}$ is the left-regular representation, and if $\mathcal{P}(A)^{-1}=\mathcal{P}\left(A^{\dagger}\right)$, or equivalently, if $\mathcal{P}(A)$ restricted to its domain and range is unitary.

Here, the left-regular representation $L_{g}: \mathbb{C}^{|G|} \rightarrow \mathbb{C}^{|G|}$ acts as $L_{g}|h\rangle=$ $|g h\rangle$. The following lemma explains why we reqire the restriction to the regular representation. 
Lemma 6.2 (Stability of isometry under concatenation). G-isometry of tensors is stable under concatenation.

Proof. $U_{g}=L_{g} \Rightarrow \Delta=\frac{1}{|G|} \mathbb{1}$, and thus the left-inverse of the concatenated tensor in 4.9 is $\left(B^{j}\right)^{\dagger}\left(A^{i}\right)^{\dagger}=\left(A^{i} B^{j}\right)^{\dagger}$ - note that the ordering of operators (as indicated by the arrows) is reversed for the layer with the inverse $P(A)^{-1}$.

Note that we do not block indices at this stage, as this would change the representation. We will show how this can be done in the section on renormalization.

\subsection{Equivalence of virtual and physical system}

The importance of $G$-isometric PEPS lies in the fact that the way the physical subspace and the virtual subspace (restricted to the $G$-invariant subspace!) are connected corresponds to a unitary, i.e., a physical operation. This implies that any unitary on the virtual level which leaves the $G$-invariant subspace invariant can be implemented by means of a unitary acting on the physical system.

Lemma 6.3. Let $A$ be a $G$-isometric PEPS. Then the unitary transformations $V$ which can be implemented on the virtual level by unitarily acting on the physical system are exactly those which commute with the symmetry, i.e., those for which

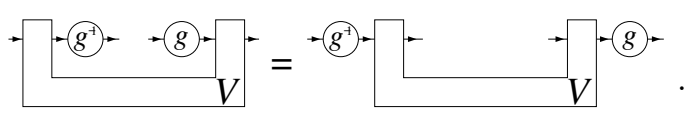

Proof. Let $V$ be a unitary which satisfies (6.1). Then, $V$ acts unitarily on the $G$-invariant subspace. Thus, the physical operation

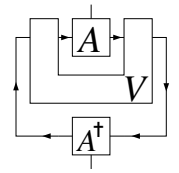

is unitary, and

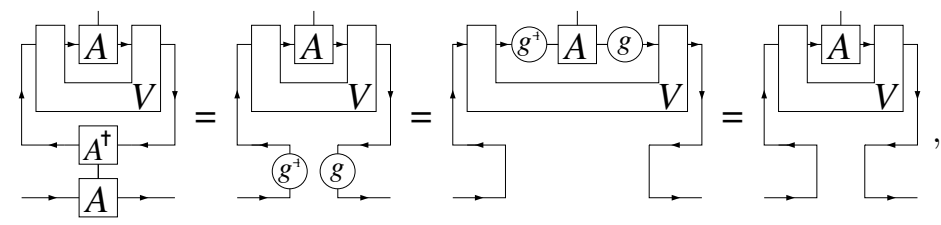

i.e., it acts as $V$ on the virtual indices. Conversely, any unitary operation $U$ on the physical level results in a $G$-invariant unitary $V=\mathcal{P}(A)^{-1} U \mathcal{P}(A)$ on the virtual level by virtue of

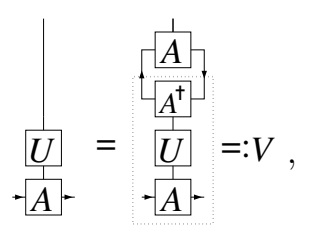


where we have used (2.4).

A particularly appealing perspective on isometric PEPS is the following.

Observation 6.4. Every $G$-isometric PEPS tensor $A$ is isomorphic to

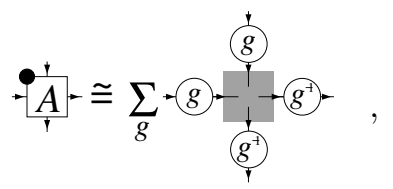

by acting unitarily on the physical system $4^{4}$ Moreover, this property is stable under concatenation. E.g.,

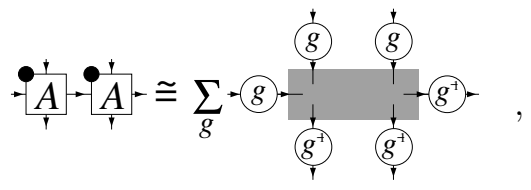

up to discarding unneeded local degrees of freedom (i.e., restricting the physical system to the subspace actually used, cf. Observation 2.4).

Eq. (6.2) follows directly from applying the left inverse (5.2), using that it is an isometry. While stability under concatenation can be inferred from Lemma 6.2 ("Isometry is stable under concatenation"), it is instructive to see how $(6.3)$ can be obtained from two tensors of the form 6.2 in a reversible way. Concatenating two tensors of the form 6.2 , we face a state of the form

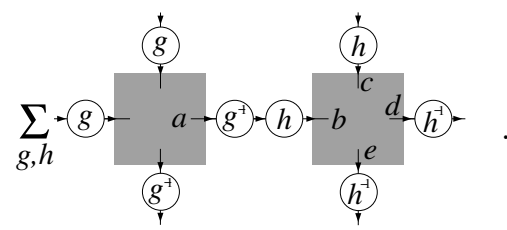

Then, we measure the two sites labeled $a$ and $b$ in the standard basis, and thus infer $g^{-1} h=a b^{-1}$. (We will use $a b$, etc. for both the site and its state.) Now, apply $U_{g^{-1} h}$ to sites $c, d$, and $e$. This maps $U_{h} \mapsto U_{h} U_{g^{-1} h}^{\dagger}=U_{g}$ (on site $c$ ) or $U_{h^{-1}} \mapsto U_{g^{-1} h} U_{h^{-1}}=U_{g^{-1}}$ (on sites $d$ and $e$, as they are bras), and thus yields a tensor of the form 6.3.

Do we loose reversibility in this process, given that we measure? No: One way to see this is to replace the measurement followed by $U_{g^{-1} h}$ by a unitary controlled by the state of $a$ and $b$, which keeps the process reversible. In particular, the state of $a$ and $b$ is separable from the rest after this operation (it is the only state which still depends on $h$ ). This, however, implies that we could equally well discard (i.e., measure) it, and rebuild it if we need to reverse the mapping.

\footnotetext{
${ }^{4}$ Note that this decomposes the physical level into four systems, represented by kets or bras depending on the directions of the arrows.
} 


\subsection{Blocking and renormalization}

An aspect which has to be taken care of separately for $G$-isometric PEPS is blocking. Recall that the proofs for the $2 \mathrm{D}$ case involved taking a block of $G$ injective tensors $A$ with representation $U_{g}$ and regarding it as a new $G$-injective 4 -index tensor $B$ with representation $V_{g}=U_{g} \otimes \cdots \otimes U_{g}$. This was possible since a tensor product of semi-regular representations is again semi-regular. In contrast, the regular case needs some special attention.

The main observation to deal with blocking in the regular case is to observe that for the left-regular representation $L_{g}$,

$$
\left(L_{g}\right)^{\otimes N} \otimes\left(L_{g}^{\dagger}\right)^{\otimes M} \cong L_{g} \otimes \mathbb{1}^{\otimes(N+M-1)} .
$$

(This follows since both representations have the same character, which means they are isomorphic.) This implies that any number of bonds with a regular representation can be mapped - on the virtual level - to only one bond carrying the $L_{g}$-symmetry, whereas all other bonds have no symmetry.

Let us now first show that this isomorphism can be implemented by a physical operation, before discussing what this implies for the structure of the PEPS. For simplicity, we restrict to the case of two bonds, $L_{g} \otimes L_{g} \cong L_{g} \otimes \mathbb{1}$. The isomorphism is implemented by the map

$$
T=\sum_{a, b}|a, a b\rangle\langle a, b|: \quad T^{\dagger}\left(L_{g} \otimes L_{g}\right) T=L_{g} \otimes \mathbb{1} .
$$

Let us now consider two tensors $A$ and $B$ adjacent to the $L_{g} \otimes L_{g}$ bond. By virtue of Observation 6.4, and grouping all other indices, they are isomorphic to

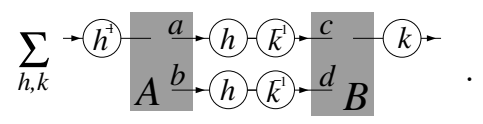

Now, we apply the unitary $T\left(T^{\dagger}\right)$ to the pair of indices labelled $a, b(c, d)$ : This physical transformation changes the network to tensors $A^{\prime}, B^{\prime}$ with symmetry $L_{g} \otimes \mathbb{1}:$

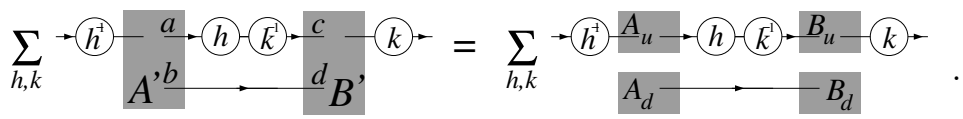

As illustrated on the r.h.s., this means that $A^{\prime}$ and $B^{\prime}$ are actually a tensor product of two independent tensors: $A \cong A_{u} \otimes A_{d}$ and $B \cong B_{u} \otimes B_{d}$. Whereas the upper tensors have an $L_{g}$-symmetry, the lower tensors have no symmetry, and form a maximally entangled pair between adjacent sites. Thus, these degrees of freedom do not contribute to the non-local properties of the PEPS, and can thus be discarded.

Observation 6.5 (Blocking indices preserves $G$-isometry). Blocking indices of $G$-isometric tensors gives a new $G$-isometric tensor, up to local isomorphisms and discarding maximally entangled pairs between adjacent sites. 
Let us now show that this procedure establishes a renormalization scheme for PEPS. The idea of renormalization is to study non-local properties of quantum many-body states by grouping several sites into one, identifying and discarding "irrelevant" (i.e., local) degrees of freedom, and iterating until convergence is reached.

Consider now a $G$-isometric PEPS with tensor $A$ (and symmetry $L_{g}$ ), and consider a block of $2 \times 2$ tensors. The blocked tensor has then symmetry $L_{g} \otimes L_{g}$, which by virtue of the above procedure can be mapped to a $L_{g} \otimes \mathbb{1}$ symmetry by local operations:

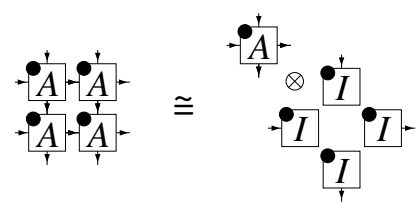

Here, the $I$ tensors are isometric tensors with no symmetry, i.e., they map the virtual system one-to-one to the physical system. Thus, by local unitaries on $2 \times 2$ blocks, the $G$-invariant PEPS with tensor $A$ has been transformed into a tensor product of the following states: First, the same PEPS described by the tensor $A$, but on a coarse-grained lattice, and second, the state given by the identity tensors $I$ - a tensor product of maximally entangled states between neighboring blocks, which can be discarded if only looking for nonlocal properties. This results in the following observation.

Observation 6.6. $G$-isometric PEPS are renormalization group fixed points 5

A similar procedure should also apply to the case of isometric tensors with a non-regular representation $U_{g}$ of the symmetry, or even to non-isometric PEPS: (Approximate) decompositions of the tensorized symmetry $U_{g}^{\otimes k}$ will yield (approximate) RG schemes for the PEPS. Another application of this RG scheme will be illustrated in the examples section: It can be used to get rid of extra symmetries in the original tensors which are of local nature and vanish after a step of blocking.

\subsection{Structure of the ground state subspace}

We will now study the properties of PEPS with a $(g, h)$-closure, Eq. (5.9), which appear as ground states of the 2D parent Hamiltonian (5.11), for the case of $G$-isometric PEPS. We want to understand three things: First, is it possible to map between arbitrary states in the ground state subspace by acting only on a restricted region? Second, are different ground states locally distinguishable? Third, what is the entropy of a continguous block of spins?

All these questions can be addressed by considering the following scenario

\footnotetext{
${ }^{5}$ Since the regular representation is the only faithful one verifying $U_{g} \otimes U_{g} \cong U_{g} \otimes \mathbb{1}, G^{-}$ isometric PEPS seem to be the unique fixed points of this type of renormalization procedure.
} 
with $G$-isometric $B$ and $D$ :

$$
\text { (b) }
$$

which by observation 6.4 is locally (where local refers to the regions $B$ and $D$ ) isomorphic to

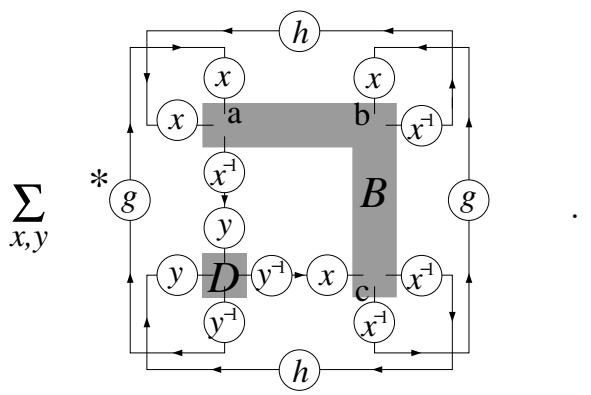

When dividing a large lattice in two blocks as above, we need to include an RG step in which we separate out $\partial D-4$ maximally entangled states between $B$ and $D$; here, the boundary $\partial D$ is the number of bonds crossing the boundary of $D$ before the RG step.

We will now devise a unitary transformation acting only on the $B$ region, which will decouple $h$ and $g$ from $D$. To this end, consider the sites marked $a, b$, and $c$ above. Since all group transformations are left regular, we have $c=x^{-1} g x b$, and thus $c b^{-1}=x^{-1} g x$ : Having access to $b$ and $c$ actually gives us access to $x^{-1} g x$. Thus, a unitary transform

$$
|c\rangle\langle b|\langle a|\mapsto| c\rangle\left\langle b\left|\left\langle c b^{-1} a|=| x^{-1} g^{-1} x a\right\rangle\right| b\right\rangle\langle c|
$$

will remove the left $g$ in 6.5 (the one marked $*$ ) from the boundary. A corresponding transformation allows us to remove the lower $h$, leaving us with

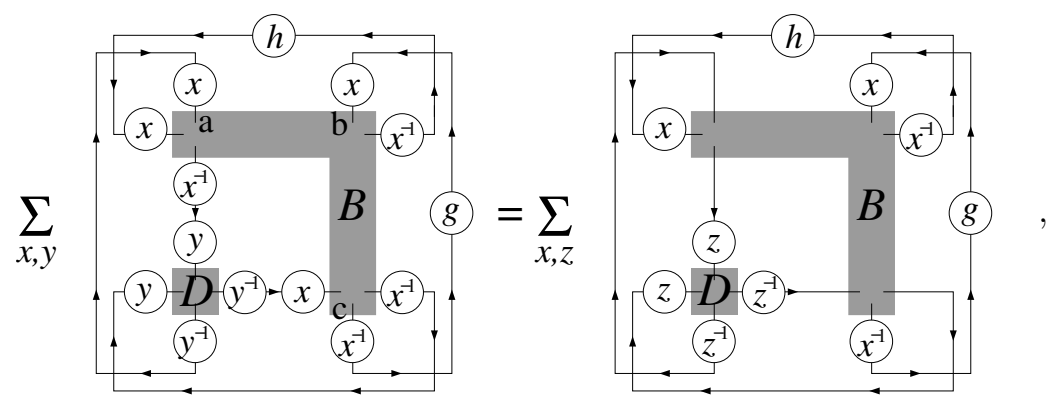

where in the second step we have substituted $z:=x^{-1} y$. Now, however, $g, h$, and $x$ only act on degrees of freedom which are completely inside the region $B$, while the degrees of freedom of $D$ are equal to the corresponding ones of $B$, 
up to a constant shift $z$. In formulas, the state above can be written (up to isomorphism) as

$$
\sum_{z}|\mu(z)\rangle_{B D}^{\otimes 4} \otimes|\zeta(g, h)\rangle_{B}
$$

with

$$
\begin{aligned}
|\mu(z)\rangle_{B D} & =\sum_{b}|z b\rangle_{B}|b\rangle_{D} \\
|\zeta(g, h)\rangle_{B} & =\sum_{x, p, q}\left|x^{-1} g x p\right\rangle_{B}|p\rangle_{B}\left|x^{-1} h x q\right\rangle_{B}|q\rangle_{B} .
\end{aligned}
$$

Note that additionally, there are $\partial D-4$ maximally entangled states between $B$ and $D$ which have been separated in the RG step.

Equation (6.7) has several immediate consequences.

Theorem 6.7 (Local equivalence of ground states). For G-isometric PEPS, it is possible to unitarily transform between any two states in the ground state subspace $\sum_{C} \lambda_{C}|\mathcal{M}(M \mid C)\rangle$ of Theorem 5.9 by acting only on two stripes (each of width one) wrapping around the torus.

Proof. By fixing two such stripes, the PEPS is partitioned as in (6.4), with the stripes labelled $B$ and the (topologically trivial) rest $D$. Then, decomposition 6.7) shows that the states $|\mathcal{M}(M \mid(g, h))\rangle$ spanning the ground state manifold differ only within $B$, allowing to unitarily transform between any two states by acting only on $B$.

Corollary 6.8 (Local indistinguishability of ground states). For G-isometric PEPS, the states in the ground state subspace of Theorem 5.9 cannot be distinguished by local operations, i.e., those which act only on a topologically trivial region.

Proof. This follows immediately by using that for any topologically trivial region $D$, it is possible to transform between any two states in the ground state subspace by acting with a unitary on its complement, $B$.

Theorem 6.9 (Topological entropy of $G$-isometric states). For a $G$-isometric $P E P S$, the reduced density operator $\rho_{D}$ of any topologically trivial block $D$ has rank $|G|^{\partial D-1}$ and a flat spectrum. Here, the length $\partial D$ of the boundary of $D$ is defined as the number of bonds crossing the boundary. Thus, both the von Neumann entropy $S$ and all Rényi entropies $S_{\alpha}$ of $\rho_{D}$ are

$$
S\left(\rho_{D}\right)=S_{\alpha}\left(\rho_{D}\right)=\log |G|(\partial D)-\log |G|,
$$

where $\log |G|$ is the topological correction to the area law $[58] 60]$.

Proof. We can again use the partitioning (6.4): We can then infer from 6.7) that (after unitary transformations on $B$ and $D$, and discarding the unentangled part of $B)$, the state is of the form $|\mu(z)\rangle^{\otimes 4}$, with

$$
|\mu(z)\rangle=\sum_{b}|z b\rangle_{B}|b\rangle_{D}
$$


Additionally, we also have $(\partial D-4)$ maximally entangled states $|\mu(1)\rangle$ from the initial RG step. It is now possible to use one copy of $|\mu(z)\rangle$ as a reference frame which allows to remove the reference to $z$ from the other copies: Take two copies of $|\mu(z)\rangle$, and apply the operation $|p, q\rangle \mapsto\left|p, p^{-1} q\right\rangle$ both on $B$ and $C$. Then,

$|\mu(z)\rangle|\mu(z)\rangle=\sum_{b c}|z b, z c\rangle_{B}|b, c\rangle_{D} \mapsto \sum_{b c}\left|z b, b^{-1} c\right\rangle_{B}\left|b, b^{-1} c\right\rangle_{D}=|\mu(z)\rangle|\mu(1)\rangle$.

Thus, all but one $|\mu(z)\rangle$ can be mapped locally to a maximally entangled state of Schmidt rank $|G|$, while the remaining "reference frame" copy - the only $z$-dependent contribution to the state - turns into $\sum_{z}|\mu(z)\rangle$ which is separable. Thus, $B$ and $D$ share a total of $(\partial D-1)$ maximally entangled states, which yields a flat Schmidt spectrum of rank $|G|^{\partial D-1} 6$

Corollary 6.10 (Topological Rényi entropy of $G$-injective states). Let $A$ be $G$ injective, with $U_{g}=L_{g}$ the regular representation. Then, any reduced density operator $\rho_{D}$ of any topologically trivial block $D$ has rank $|G|^{\partial D-1}$, and thus its zero Rényi entropy is

$$
S_{0}\left(\rho_{D}\right)=\log |G|(\partial D)-\log |G|
$$

Proof. This follows immediately from the preceding theorem, since any $G$-injective $A$ with $U_{g}=L_{g}$ can be transformed to a $G$-isometric one by a local (nonunitary) transformation on the physical system. (This can be seen by doing a singular value decomposition of $\mathcal{P}(A)=U D V: U D$ is the desired linear operation, which by Observation 2.4 has full rank.)

\subsection{Commuting parent Hamiltonians}

Let us now show that the parent Hamiltonians of $G$-isometric PEPS are special: They are sums of commuting local terms. We will demonstrate the proof for MPS, but the generalization to PEPS is straightforward.

Lemma 6.11. For $G$-isometric MPS, the local terms $h_{i}[E q$. 5.10]] in the parent Hamiltonian - which project onto the complement of $\mathcal{S}_{2}=\left\{\sum_{i j} \operatorname{tr}\left[A^{i} A^{j} X\right]|i j\rangle \mid X\right\}$ - are of the form

$$
\text { . }
$$

\footnotetext{
6 Note that we could have used the same argument without the initial RG step: In that case, we would have arrived at $\partial D$ copies of $|\mu(z)\rangle$ which could have been converted to $(\partial D-1)$ maximally entangled states the same way.
} 
Proof. First, $h_{i}$ is a projector, since

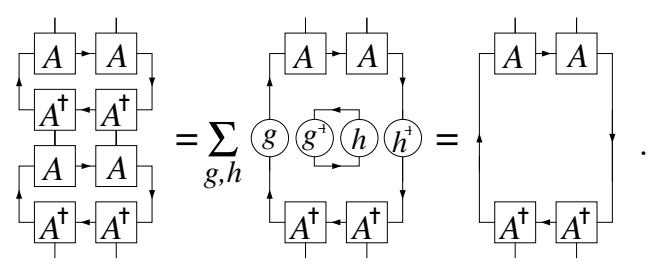

Second, its range is clearly contained in $\mathcal{S}_{2}$, and third, any state of the form $\sum_{i j} \operatorname{tr}\left[A^{i} A^{j} X\right]$ is preserved by $h_{i}$ :

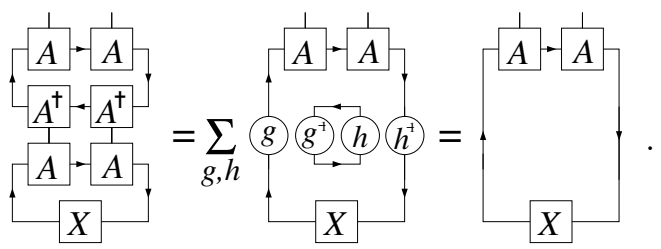

Finally, $h_{i}$ is self-adjoint, which proves the lemma.

Note that the corresponding operator (6.8) defined for non-isometric PEPS (with $A^{-1}$ instead of $A^{\dagger}$ ) fails the last condition: while it is a projector onto $\mathcal{S}_{2}$, it is not self-adjoint, i.e., not a Hamiltonian.

Theorem 6.12 (Commuting Parent Hamiltonians). For G-isometric PEPS, the terms $h_{i}$ of the parent Hamiltonian of Theorem 5.7 cf. (6.8), commute.

Proof. We start from the identity

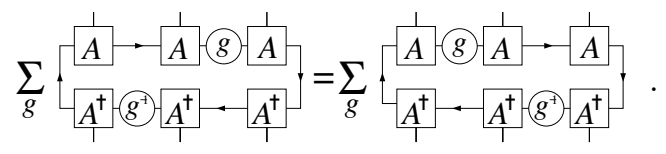

(Note that this the projector onto the joint kernel of $h_{1}$ and $h_{2}$, which is equal $h_{1} h_{2}$ for commuting $h_{1}, h_{2}$ !) The l.h.s. can be transformed to

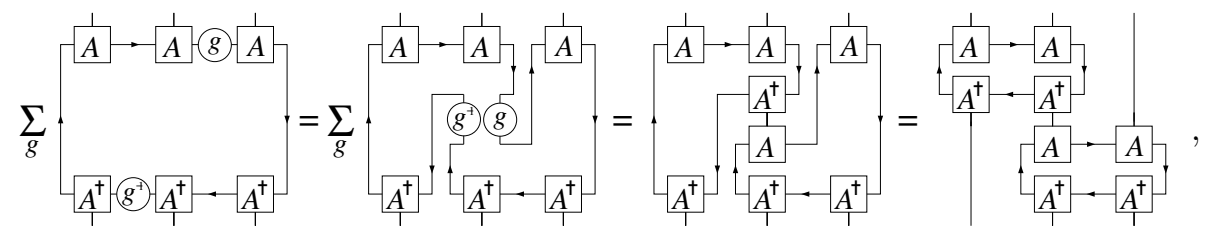

i.e., $h_{1} h_{2}$, while the corresponding transformation on the r.h.s. yields $h_{2} h_{1}$, proving that $h_{1} h_{2}=h_{2} h_{1}$. The same proof applies to two dimensions. 


\subsection{Anyons}

In the following, we show how to understand anyonic excitations of $G$-isometric PEPS using the symmetry of the tensors. Intuitively, excitations should be formed by (open) strings of $U_{g}$ 's, similar to the (closed) strings distinguishing the different ground states: Since such strings can be continuously deformed, they cannot be detectable anywhere locally, except for the endpoints. As we will show, these strings give rise to one type of particles: fluxes, but there will also be a second type of complementary particles: charges.

Note that in the following, we consider the bulk of a PEPS formed by $G^{-}$ isometric tensors $A$, and assume the boundaries to be far away: The results generally hold independent of the form of the boundaries.

\section{Magnetic fluxes}

Definition 6.13 (Fluxons). Consider a PEPS formed of $G$-isometric tensors $A$, let $g \in G$, and let $U_{g}=L_{g}$ be the left-regular representation. A pair of magnetic fluxes or fluxons in the state $\left(g, g^{-1}\right)$ is defined by placing a string of $U_{g}$ and $U_{g^{-1}}$ on the virtual level as follows:

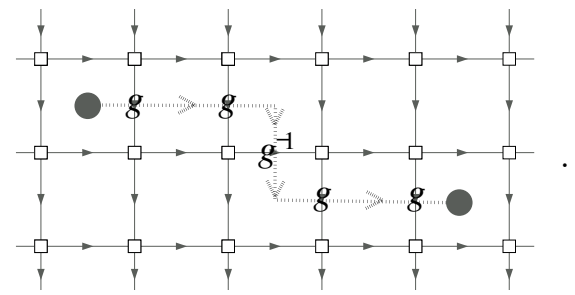

The fluxons are attached to the two marked plaquettes forming the endpoints of the string; they are characterized by the fact that there is an odd number of $g$ and $g^{-1}$ on the adjacent edges. Whether $g$ or $g^{-1}$ is used depends on the orientation of the string relative to the bonds; we will generally talk of a "string of $g$ 's" meaning both $g$ and $g^{-1}$. Also, we assign the state $g$ to the starting point and $g^{-1}$ to the endpoint. The particle type of the two fluxons is given by the conjugacy classes $C[g]$ and $C\left[g^{-1}\right]$, while the exact states $g$ and $g^{-1}$ determine the internal state of the fluxons. We will show in the following why these choices make sense.

Lemma 6.14 (Deformations of the string). The endpoint plaquettes of a string are fixed. Except for that, the string connecting a pair of fluxons can be deformed at will without acting on the system. This implies that it cannot be observed anywhere except for the endpoints.

Proof. The string can be defomed using the $G$-invariance of the tensors, cf. (5.13), as long as the endpoints are not involved. On the other hand, the $G$-invariance cannot change the parity of $g$ 's around a plaquette, which implies that the endpoints cannot be moved. (However, the string can be deformed to reach the endpoint from any side.) Since according to the following theorem, endpoints 
can be measured, there also cannot be any other way to move them away without acting on the physical system.

Theorem 6.15 (Detection of fluxons). The particle type $C[g]$ of a fluxon can be detected by a measurement on the plaquette supporting it.

Proof. As we can arbitrarily deform the string of $g$ 's ending at a given plaquette, it suffices to consider the following situation:

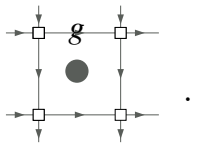

We will use the four sites of the plaquette to build a "tweezer" which allows us to "grab" $g$, up to a twirl. To this end, block the four sites to a U-shaped tensor. From Observation 6.4 we know that there is a reversible operation which transforms the blocked tensor to

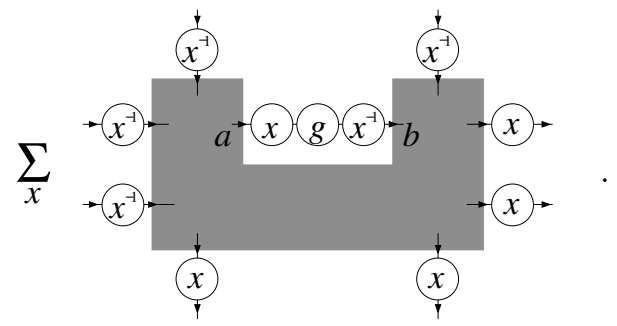

By measuring $a b^{-1}=x g x^{-1}$, we can now infer the particle type $C[g]$ of the fluxon. Since the value of $x$ cannot be determined, this is all the information we can gain about a single fluxon.

Looking at (6.11) and the way our measurement of $C[g]$ works, one can see that we actually do not use any of the outgoing indices, i.e., those which connect to the virtual level and thus to neighboring tensors. Therefore, we can simplify notation by omitting these indices together with the sum over $x$ and analyze the action of the detection tweezer on

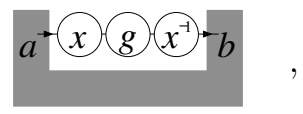

where $x$ is unknown. We will make use of this simplified notation from now on in the analysis of subsequent constructions for detecting, moving, and creating anyons.

Note that the reason we built a tweezer and not just acted on the site left and right of $g$ in $(6.10)$ is that we needed to synchronize the twirl; otherwise, we could have only determined $x g y^{-1}$, which is completely random. Let us also note that the tweezer can open in any direction, as the string of $g$ 's can be attached to any egde of the plaquette. 
Theorem 6.16 (Moving of fluxons). Fluxons can be moved by local unitaries, without knowledge of their state.

Proof. We give the construction for the scenario where we want to move a string to the right, i.e., achieve the following transformation

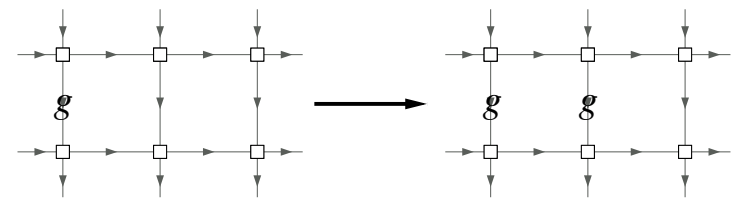

by means of local unitaries. To this end, we will again build a tweezer (opening towards the left) which will allow us to access the two vertical edges on the left. Again, using Observation 6.4 and neglecting unneeded bonds, the above transformation is equivalent to the transformation

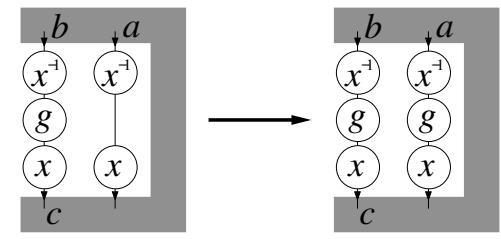

for arbitrary $x$. This can be accomplished using the same transformation already used in (6.6) for this purpose: $|a\rangle|b\rangle\left\langle c|\mapsto| b c^{-1} a\right\rangle|b\rangle\langle c|$. (Note that each of the kets and bras describes the state of a separate quantum system: $|c\rangle\langle a|\langle b|$ thus describes a pure state of the three systems $a, b$, and $c$. Whether we use kets or bras is determined by the arrows associated to the links.)

Theorem 6.17 (Creation of fluxons). For any $g \in G$, the fluxon pair

$$
\sum_{z}\left|\left(z g z^{-1}, z g^{-1} z^{-1}\right)\right\rangle
$$

can be created deterministically by local operations 7 Note there is one such state per conjugacy class $C[g]$.

Proof. We want to implement the transformation

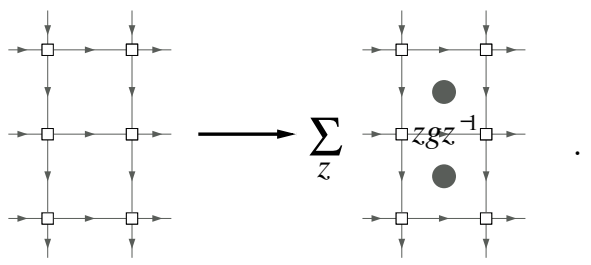

7 The reason that we can only create the equal weight superposition is that all other superpositions carry non-zero charge, which is a conserved quantity, cf. 57. 
To this end, we build a tweezer on the lower plaquette. Neglecting unneeded degrees of freedom, the transformation we want to achieve is equivalent to

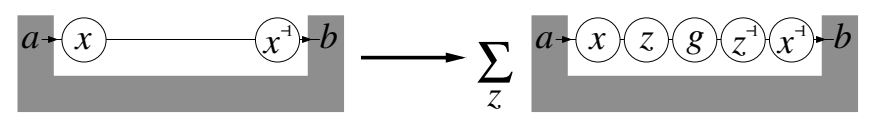

for some unknown $x$. This can be accomplished by replacing the state $\sum_{r}|r\rangle_{a}\left\langle\left. r\right|_{b}\right.$ with $\sum_{s, z}\left|z g z^{-1} s\right\rangle_{a}\left\langle\left. s\right|_{b}\right.$, which can be done unitarily if wanted.

\section{Braiding of fluxons}

In the following, we investigate what happens to fluxons when they are braided around each other. The general scenario is as follows: Consider two pairs of fluxons, and move one fluxon of one pair around one of the other one:

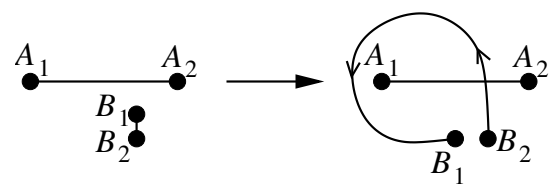

In principle, it does not make sense to ask how the anyon $B_{1}$ is affected when it crosses the line between $A_{1}$ and $A_{2}$, since this line can be deformed at will. However, it does make sense to think like that if the pair $B_{1}$ and $B_{2}$ is brought together after braiding to measure the joint flux, since then $B_{1}$ eventually has to cross this line. We will discuss how to measure the joint flux later on.

Instead of investigating what happens when we move the fluxon $B_{1}$ across the string connecting $A_{1}$ and $A_{2}$, we rather keep $B_{1}$ fixed and move the string across it - this is easier to analyze, since it takes place purely on the virtual level:

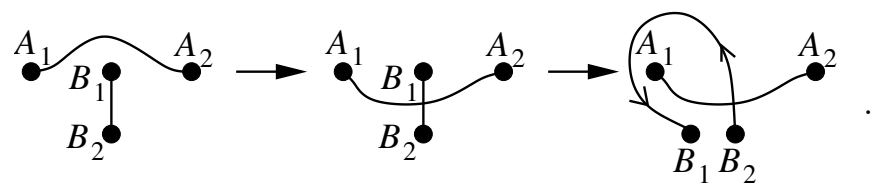

Let us now consider this scenario - moving the $A_{1}-A_{2}$ string accross the anyon $B_{1}$ - in the PEPS representation on the virtual level. To this end, let $A_{1}$ and $A_{2}$ be in the state $\left(g, g^{-1}\right)$, and $B_{1}$ and $B_{2}$ in state $\left(h, h^{-1}\right)$. Then, using the $G$-invariance of the tensors,

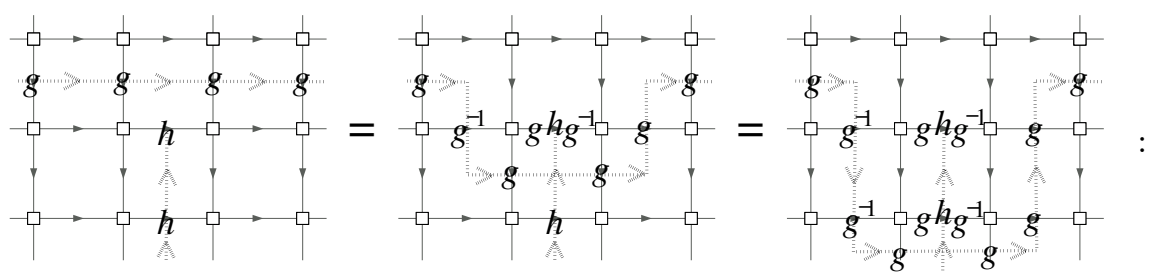


i.e., the $B_{1}$ end of the string is conjugated by $g$, and is now in the state $g h g^{-1}$. Note that this does not change the conjugacy class, i.e., the particle type. This is crucial since the crossing of $B_{1}$ and the $A_{1}-A_{2}$ line cannot be assigned a welldefined position, and thus the change of the state of $B_{2}$ cannot be detected by measuring $B_{2}$ alone.

However, when we bring $B_{1}$ and $B_{2}$ together, it is possible to measure the particle type $C\left[g h g^{-1} h^{-1}\right]$ of their joint flux, and thus infer information about the state $g$ of $A_{1}$. For two fluxes in states $a$ and $b$, their joint state is given by $a b$; this is consistent with how the two fluxes together affect another flux when braiding. Note that actually merging the two fluxes will require a measurement, since this is an irreversible process.

Let us now describe how to measure the joint flux. To this end, consider two fluxes $a$ and $b$ sitting on adjacent plaquettes - this is as close as we can bring them using the "fluxon moving tweezer" of Theorem 6.16 - and route the virtual strings as follows:

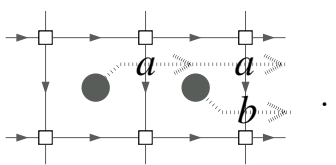

Now, we can use basically the same tweezer construction used for measuring single fluxons in Theorem 6.15 to measure the $C[a b]$ : To this end, we build a tweezer covering both plaquettes, and connected at the left side, and measure $C[a b]$ across the right vertex. In fact, this construction can be extended to measure the joint flux of any number of anyons enclosed in the tweezer.

Note that the braiding (6.14) does not only change the state of $B_{1}$, but also that of $A_{1}$ if it brought back to interfere with $A_{2}$. The reason is that even after repairing the $B$ particles, the loop of $h$ 's remains there and affects any anyon crossing it. Note that while the way $A_{1}$ is affected when moving it out of the loop depends on whether it is moved above or below the path it came from, this difference cannot be observed when repairing it with $A_{2}$, and vanishes when $A_{1}$ and $A_{2}$ are aligned in a fixed way.

\section{Electric charges}

Let us now define electric charges. Different from fluxes which always come in pairs, it is possible to define (though not create) electric monopoles.

Definition 6.18 (Electric charges). An electric charge ("chargeon") with charge $c$, where $c$ labels an irreducible representation $D^{c}$ of $G$, is given by a defect

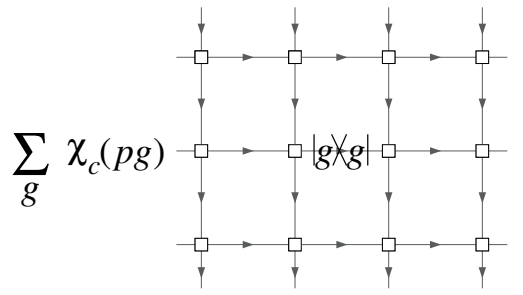


which is attached to an edge of the lattice, i.e., instead of the identity, the edge acts as $\sum_{g} \chi_{g}(p g)|g\rangle\langle g|$. Here, $\chi_{c}(\cdot)=\operatorname{tr}\left[D^{c}(\cdot)\right]$ is the character of the irreducible representation $c$, and $p \in G$ characterizes the internal state of the chargeon.

Theorem 6.19 (Detection of chargeons). The charge $c$ of a chargeon can be detected by measuring across the two vertices adjacent to the edge supporting the charge.

Note that we will later show that the internal degree of freedom $p$ is changed by strings connecting fluxons, which rules out that we can measure $p$ itself.

Proof. We will perform a joint measurement on the vertices left and right of the chargeon in 6.15). From Observation 6.4. the state of these two sites is isomorphic (up to unneeded links) to

$$
\sum_{g} \chi_{c}(p g)+x-g \nmid g \mid \vartheta
$$

i.e., we have access to the state

$$
\sum_{g} \chi_{c}(p g)|x g\rangle\left\langle y g\left|=\sum_{k} \chi_{c}\left(p x^{-1} k\right)\right| k\right\rangle\langle z k|
$$

for some $x$ and $y$, with $k:=x g$ and $z=y x^{-1}$. As a consequence of the group orthogonality relations, $\left|v_{h}^{c}\right\rangle=\sum_{k} \chi_{c}(h k)|k\rangle$ are orthogonal vectors for different irreducible representations $c,\left\langle v_{h}^{c} \mid v_{h^{\prime}}^{c^{\prime}}\right\rangle=f\left(h, h^{\prime}\right) \delta_{c, c^{\prime}}$. Therefore, the charge $c$ (as well as $z$ ) can be determined by measuring the two sites in (6.16). Note that on the other hand, no information about $p$ can be learned, since $x$ is unkown.

Theorem 6.20 (Moving of chargeons). Chargeons can be moved by local operations.

Proof. Since chargeons are localized perturbations on the virtual level (unlike fluxons which arise from strings), moving then is as simple as swapping: In order to implement the transformation

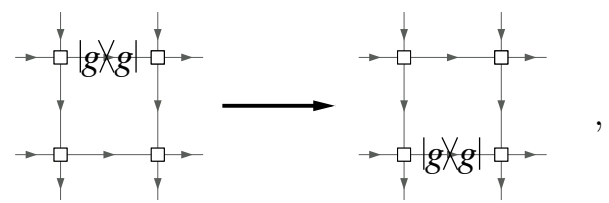

we will need to block the left two and right two spins independently. The transformation is then locally equivalent (up to unneeded degrees of freedom) to

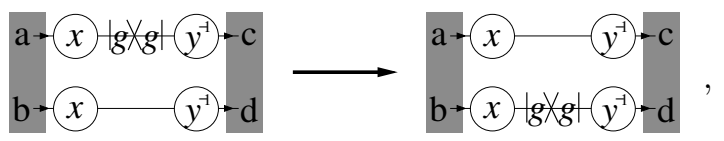


and thus, by swapping a with b and $\mathrm{c}$ with $\mathrm{d}$, the chargeon is moved as in (6.17). Note that a similar construction can also be used to move a fluxon around the corner.

Theorem 6.21 (Creation of chargeons). For any irreducible representation $c$ and any $p \in G$, the chargeon pair described by

$$
\Pi_{c, p}=\sum_{g, h} \chi_{c}\left(p h^{-1} g\right)|g\rangle\left\langle\left. g\right|_{u} \otimes \mid h\right\rangle\left\langle\left. h\right|_{d}\right.
$$

can be created by local operations.

Note that this gives a particle with charge $c$ in position $u$ and a particle of charge $\bar{c}$ in position $d$, where $\bar{c}$ labels the complex conjugate of representation $c$.

Proof. First, observe that the pair 6.18 is invariant under conjugation by $x \in$ $G$ :

$$
\begin{aligned}
\left(U_{x} \otimes U_{x}\right) \Pi_{c, p}\left(U_{x}^{\dagger} \otimes U_{x}^{\dagger}\right) & =\sum_{g, h} \chi_{c}\left(p h^{-1} g\right)|x g\rangle\langle x g|\otimes| x h\rangle\langle x h| \\
& =\sum_{\tilde{g}, \tilde{h}} \chi_{c}\left(p \tilde{h}^{-1} x x^{-1} \tilde{g}\right)|\tilde{g}\rangle\langle\tilde{g}|\otimes| \tilde{h}\rangle\langle\tilde{h}|=\Pi_{c, p} .
\end{aligned}
$$

To create the state 6.18 , we need to implement the transformation

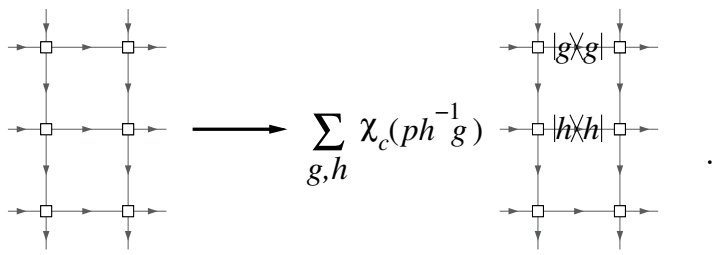

To this end, we use a U-shaped tweezer encompassing all six sites, which leaves us with the task of implementing the transformation

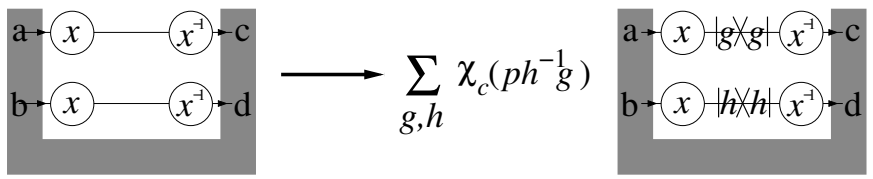

for some unkown $x$. Due to the $U_{x}$-invariance of $\Pi_{c, p}[\mathrm{Eq} .66 .19]$, neither side depends on $x$, and the transformation can be implemented (unitarily) by mapping the initial state of the four sites a,b,c,d to $\Pi_{c, p}$. 


\section{Braiding of charges with fluxes}

Let us finally see what happens when we braid chargeons with other particles. Since chargeons are localized defects in the lattice, they do not affect other particles which are braided around them. On the other hand, they are affected if braided around a fluxon. Consider again the braiding (6.14), now with $A_{1}-A_{2}$ a pair of fluxons in state $\left(k, k^{-1}\right)$, and $B_{1}-B_{2}$ a pair of chargeons with charges $c$ and $\bar{c}$, e.g. as in 6.18). Again, we will choose to move the string connecting the fluxons $A_{1}$ and $A_{2}$ over the chargeon $B_{1}$, rather than moving $B_{1}$ across the string. Analyzing the situation for a single projection $|g\rangle\langle g|$, we find that

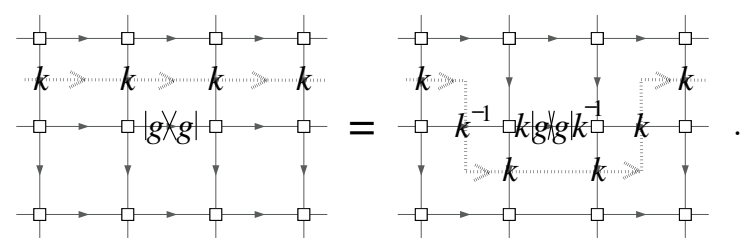

This is, $|g\rangle\left\langle g\left|\mapsto U_{k}\right| g\right\rangle\left\langle g\left|U_{k}^{\dagger}=\right| k g\right\rangle\langle k g|$, and thus

$$
\sum_{g} \chi_{c}(p g)|g\rangle\left\langle g\left|\mapsto \sum_{\tilde{g}} \chi_{c}\left(p k^{-1} \tilde{g}\right)\right| \tilde{g}\right\rangle\langle\tilde{g}|:
$$

braiding a chargeon around a fluxon of flux $k$ leaves its type $c$ invariant, but multiplies the parameter $p$ characterizing its internal state by $k^{-1}$.

To interferometrically detect this change, one can e.g. start from a pair of charges in state $\Pi_{c, p}(6.18)$, braid them around the flux $k$, and bring them back together. Then one can reason as follows. We have seen that, by acting with a unitary $W$ on six sites, the original state (before the braiding) decomposes as $\sum_{x}\left|\Pi_{c, p}\right\rangle_{a b c d}\left|\psi_{x}\right\rangle$ where $|G|\left|\Pi_{c, p}\right\rangle_{a b c d}$ is exactly the right hand side of 6 6.20 which is indeed independent of $x$. The group orthogonality relations assure that $\left|\Pi_{c, p}\right\rangle_{a b c d}$ is normalized, which in turn gives $\sum_{x, y}\left\langle\psi_{x} \mid \psi_{y}\right\rangle=1$. Now, after braiding and applying $W$ we are left with $\sum_{x}\left|\Pi_{c, p, k}^{x}\right\rangle_{a b c d}\left|\psi_{x}\right\rangle$, where $|G|\left|\Pi_{c, p, k}^{x}\right\rangle_{a b c d}$ is the right hand side of 6.20 but with the coefficients given by $\chi_{c}\left(p h^{-1} k^{-1} g\right)$. Then, we can implement in abcd (after having applied $W$ ) the measurement given by $\left|\Pi_{c, p}\right\rangle\left\langle\left.\Pi_{c, p}\right|_{a b c d}\right.$ and its orthogonal complement, which outputs that the state is unchanged with probability

$$
\left|\frac{\chi_{c}(k)}{|c|}\right|^{2}
$$

where $|c|$ denotes the dimension of the representation. To see that, it is enough to use the group orthogonality relations once more.

\section{$7 \quad$ Examples}

In the following, we present examples of $G$-injective PEPS. We start by discussing Kitaev's code state [56], including an explanation of how to construct 


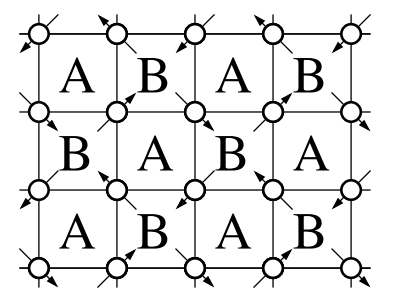

Figure 1: The lattice for Kitaev's code state and the double models.

the original PEPS and to obtain the $G$-isometric PEPS by an RG step. We then consider the generalization of the model to arbitrary finite groups [56], the so-called double models, and show that they correspond to the set of $G$-isometric PEPS. Finally, we show that by using semi-regular representations, it is possible to construct PEPS which are locally equivalent to the double models, yet can be realized with a lower bond dimension.

\subsection{Kitaev's code state}

Kitaev's code state can be defined on a square lattice with qubits $\{|0\rangle,|1\rangle\}$ attached to its vertices, and two types of plaquettes, A and B, see Fig. 1. (The arrows will be used in the non-abelian case). The Hamiltonian consists of local terms associated to plaquettes, each acting on the four surrounding qubits: For each A plaquette $p_{A}$, define

$$
h_{A}^{p_{A}}:=\frac{1}{2}\left(1-Z^{\otimes 4}\right)
$$

acting on the four sites adjacent to the plaquette $p_{A}$; this is, $h_{A}^{p_{A}}$ is the projector whose ground state subspace is formed by the configurations $|g, h, k, l\rangle$ with even parity around the plaquette. For each B plaquette $p_{B}$, define

$$
\begin{aligned}
h_{B}^{p_{B}} & :=\frac{1}{2}\left(1-X^{\otimes 4}\right) \\
& =\sum_{s}|g+s, h+s, k+s, l+s\rangle\langle g, h, k, l|,
\end{aligned}
$$

where addition is modulo 2 ; this is, $h_{B}^{p_{B}}$ is a projector whose ground state subspace is invariant under flipping all spins adjacent to $p_{B}$. Now let

$$
H_{A}:=\sum_{p_{A}} h_{A}^{p_{A}}, \text { and } H_{B}:=\sum_{p_{B}} h_{B}^{p_{B}} .
$$

Then, Kitaev's code state Hamiltonian [56 is given by $H=H_{A}+H_{B}$.

Let us now explain how to construct a PEPS representation of a ground state of $H$ [44. To this end, observe that all terms $h_{A}^{p_{A}}, h_{B}^{p_{B}}$ in the Hamiltonian commute and are projections: Thus, the ground state subspace is given by the product

$$
\prod_{p_{B}} \frac{1}{2}\left(1-h_{B}^{p_{B}}\right) \prod_{p_{A}} \frac{1}{2}\left(1-h_{A}^{p_{A}}\right)
$$


and a product of local operators can always be written as a PEPS.

A particularly appealing way to construct the PEPS representation is the following. We start from the state $|0, \ldots, 0\rangle$, which has even parity around each plaquette and is thus a ground state of $H_{A}$. By sequentially applying all projections $\frac{1}{2}\left(1-h_{B}^{p_{B}}\right)$ to the initial $|0, \ldots, 0\rangle$ state, we thus end up with a ground state of $H{ }^{8}$

The projector $\frac{1}{2}\left(1-h_{B}^{p_{B}}\right)$ can be expressed as a tensor network of the form

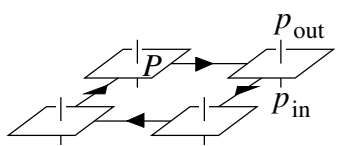

which takes a layer of physical inputs $p_{\text {in }}$ at the bottom, and maps them to a new layer of physical outputs $p_{\text {out }}$ at the top. The four PEPS tensors above are all equal,

$$
P=\sum_{s, k}|k+s\rangle_{p_{\text {out }}}\left\langle\left. k\right|_{p_{\text {in }}} \otimes \mid s\right\rangle_{v}\left\langle\left. s\right|_{v}\right.
$$

where $v$ denotes the virtual indices. The natural way to think of this construction is to assign the value $s$ to the plaquette rather than to the bonds: When contracting the virtual indices around the loop, there only remains a single sum over $s$, which implements the projection $\frac{1}{2}\left(1-h_{B}^{p_{B}}\right)$ by simulateously flipping all four physical spins conditionally on $s$.

The B plaquette projections can be divided into two disjoint layers, the PEPS representation of each of which is given by 7.3 . When combining the layers, at each site two $P$ tensors $(7.3$ are stacked atop of each other, with the virtual indices pointing in opposite directions. In order to obtain the PEPS description of the ground state itself, we have to apply these two layers of $P$ to the initial state $|0\rangle$. This yields the tensor (cf. [44])

$$
\begin{aligned}
T & =\sum_{r, s}|r+s\rangle_{p}|r, s\rangle_{v}\left\langle r,\left.s\right|_{v}\right. \\
& =|0\rangle\left[|0,0\rangle_{v}\left\langle 0,\left.0\right|_{v}+\mid 1,1\right\rangle_{v}\left\langle 1,\left.1\right|_{v}\right]+|1\rangle\left[|0,1\rangle_{v}\left\langle 0,\left.1\right|_{v}+\mid 1,0\right\rangle_{v}\left\langle 1,\left.0\right|_{v}\right]\right.\right.
\end{aligned}
$$

Here, the $r$ 's and $s$ 's on the virtual level are placed such that they are associated with one B plaquette each,

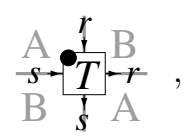

and correspondingly rotated for the other type of vertices.

It is again convenient to think of the virtual indices as assigned to B plaquettes, rather than to bonds. Then, the value of each qubit in the lattice is given

\footnotetext{
8 Note we have to make sure that $|0, \ldots, 0\rangle$ has non-zero overlap with the ground state subspace of $H_{B}$. This is true as it has non-zero overlap with $(|0\rangle+|1\rangle) \otimes \cdots \otimes(|0\rangle+|1\rangle)$ which is in the kernel of $H_{B}$.
} 
by the difference $r-s \equiv r+s$ between the virtual degrees of freedom $r$ and $s$ assigned to the two adjacent B plaquettes. Alternatively, one can think of the state as arising from $|0, \ldots, 0\rangle$ - an eigenstate of $H_{A}$ - which is symmetrized by coherently flipping the spins adjacent to any B plaquette, controlled by its virtual degree of freedom.

From (7.4), it is straightforward to see that $T$ is characterized by the following symmetries:

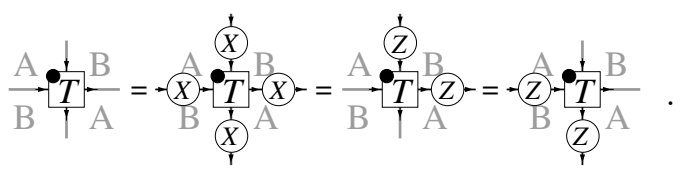

Thus, $T$ has the desired $\mathbb{Z}_{2}$-invariance with respect to $X^{\otimes 4}$, but it is lacking $\mathbb{Z}_{2}$-injectivity since it has additional symmetries with respect to $Z^{\otimes 2}$. In order to get rid of these symmetries, we consider a $2 \times 2$ block around an A plaquette, which has symmetries

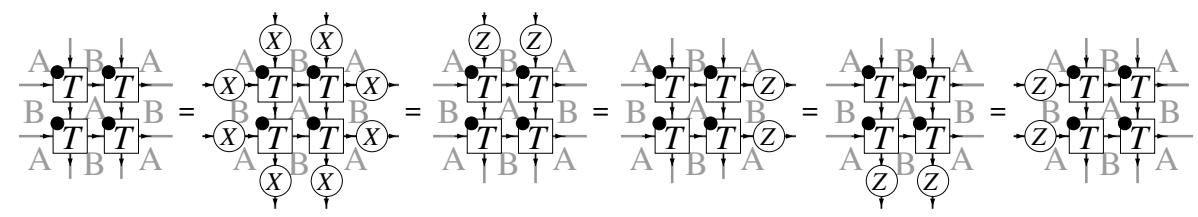

The $X^{\otimes 8}$ symmetry is the topologically interesting one, whereas the $Z^{\otimes 2}$ symmetries, after blocking, only act between adjacent tensors: They should therefore be local in nature. Moreover, since $X \otimes X$ and $Z \otimes Z$ commute, there has to be an isometry which separates their action: This is accomplished by the CNOT gate $|a\rangle|b\rangle \mapsto|a\rangle|a+b\rangle$ which we have already used for the RG flow in Sec. 6.3, and which maps

$$
\begin{aligned}
X \otimes X & \mapsto X \otimes \mathbb{1} \\
Z \otimes Z & \mapsto \mathbb{1} \otimes Z .
\end{aligned}
$$

By applying these CNOTs, the $2 \times 2$-block of $T$ 's is thus transformed tq 9

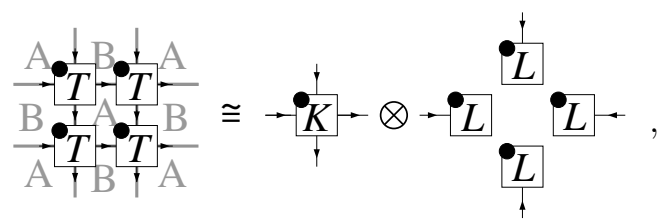

where the tensors $K$ and $L$ have exactly the symmetries

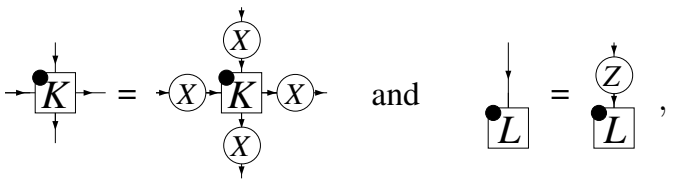

${ }^{9}$ Note that since $\mathcal{P}(T)$ is isometric on its support, the RG step can be implemented by a physical unitary and a relabelling of bonds. 
respectively. Thus, the tensor $K$ is the desired $\mathbb{Z}_{2}$-isometric tensor describing the topological code state, while the $L$ are tensors with only one virtual qubit index with $\mathbb{Z}_{2}$ symmetry: They therefore give rise to product states between adjacent sites. Note that this also explains why the original PEPS representation of the code state needed double as many bonds as actually required to obtain the topological entropy: The original tensor $T$ has extra local symmetries.

Let us now try to see how the blocked and renormalized tensors $K$ actually look like. As compared to the original lattice, the tensors sit on top of (i.e., contain) an A plaquette, and each bond corresponds to a B plaquette. The other half of the A plaquettes forms the plaquettes between the renormalized tensors:

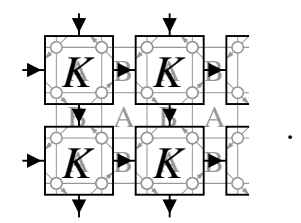

The summation index $s$ in the PEPS construction 7.3 and (7.4), which we argued was associated to B plaquettes, is now associated to edges, i.e., it became an actual bond state. Going through the RG scheme (or observing that the action of the tensor $P$, Eq. (7.3), is to add $s$ to all qubits surrounding the associated B plaquette), we find that

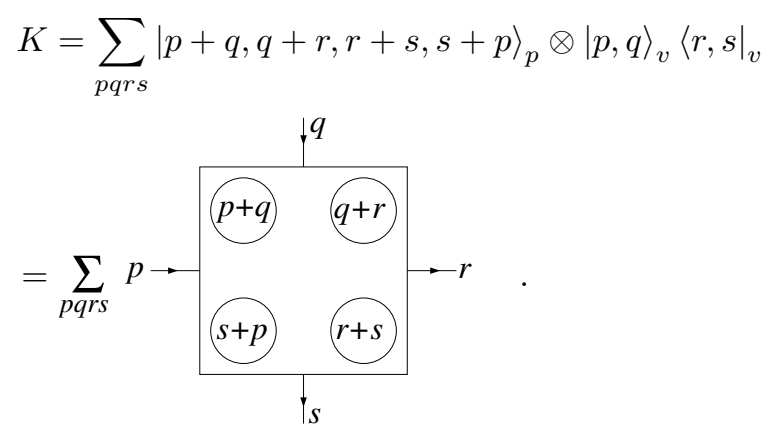

One possible perspective on the PEPS representation is thus to assign values 0 or 1 ("colors") to each $B$ plaquette, and let the physical states be the difference between the colors of the adjacent plaquettes; the state is then given by the superposition over all color patterns. Note that $\mathcal{P}(K)$ does not have full rank, as the physical subspace in 7.5 is restricted to states with even parity.

What is the parent Hamiltonian for the blocked tensor? As it turns out, it can be split into three components, each of which can be associated to a term in the original Hamiltonian: i) a local term which enforces the local constraint; ii) a four-site term enforcing the plaquette constraint for the A plaquette covering four tensors; and iii) a two-site term making sure that the state is an equal weight superposition of all edge colorings: it enforces that the state is invariant under adding 1 to all four qubits surrounding the edge. 
As we have seen earlier, there is also a different way to express the PEPS tensor $K$, using Observation 6.4

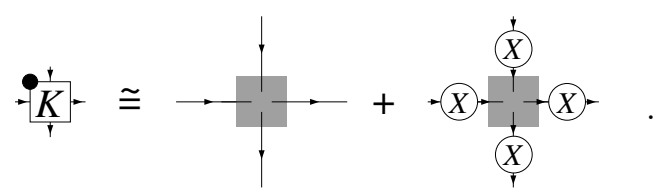

Written in this form, the four physical qubits are +1 eigenstates of $X^{\otimes 4}$, rather than $Z^{\otimes 4}$ as in $(7.5)$, and the different terms in the Hamiltonian ensure i) that this local constraint is obeyed, ii) that the product of twirls around a plaquette vanishes, and iii) that all possible twirls on an edge appear with equal weight.

\subsection{The double models}

Kitaev's code state Hamiltonian can be naturally generalized to any finite group $G$. To this end, identify the states at each site with group elements $|g\rangle$, and generalize the Hamiltonian terms as follows:

$$
h_{A}^{p_{A}}:=1-\sum_{g h k l=1}|g, h, k, l\rangle\langle g, h, k, l|,
$$

where the sum is over all group elements whose product $g h k l$ equals the identity 1 , and the product is taken in the order indicated by the arrows in Fig. 1 from left to right; and

$$
h_{B}^{p_{B}}:=1-\sum_{s}\left|U_{s} g, U_{s} h, U_{s} k, U_{s} l\right\rangle\langle g, h, k, l|,
$$

where $U_{s}=L_{s}\left(U_{s}=R_{s}\right)$ if in Fig. 1, the arrow on the spin points away from (towards) $p_{B}$; we keep this convention throughout. Here, $L_{s}:|s\rangle \mapsto|s g\rangle$ and $R_{s}:|s\rangle \mapsto\left|g s^{-1}\right\rangle$ are the left and right multiplication, respectively. The total Hamiltonian is again the sum of these terms for all plaquettes.

The PEPS construction can be carried out the same way as for the $\mathbb{Z}_{2}$ case, by starting from $|1, \ldots, 1\rangle$ and applying projectors

$$
P=\sum_{s, k} U_{s}|k\rangle_{p_{\text {out }}}\left\langle\left. k\right|_{p_{\text {in }}} \otimes \mid s\right\rangle_{v}\left\langle\left. s\right|_{v} ;\right.
$$

applying two of these projectors to $|1\rangle$, we arrive at the PEPS tensor

$$
T=\sum_{r, s} U_{r} U_{s}|1\rangle_{p}|r, s\rangle_{v}\left\langle r,\left.s\right|_{v} .\right.
$$

Here, the action of $U_{r}$ and $U_{s}$ is determined by the site it acts on, and by the plaquette associated with $r$ and $s$, respectively (which implies the order does not matter); $U_{r} U_{s}|1\rangle$ is thus either $\left|r s^{-1}\right\rangle$ or $\left|s r^{-1}\right\rangle$. 
The tensor $T$ has again additional symmetries, which can be removed by blocking around an A plaquette $\left.\right|^{10}$ and renormalizing via $|a\rangle|b\rangle \mapsto|a\rangle\left|b^{-1} a\right\rangle$; this yields the $G$-isometric tensor for the quantum double,

$$
\begin{aligned}
& K=\sum\left|p q^{-1}, q r^{-1}, r s^{-1}, s p^{-1}\right\rangle_{p} \otimes|p, q\rangle_{v}\left\langle r,\left.s\right|_{v}\right.
\end{aligned}
$$

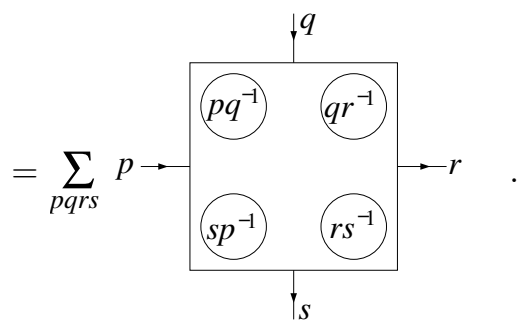

Note that this PEPS representation can again be interpreted by assigning colors, i.e., group elements, to the bonds, and letting the state of each qu- $|G|$-it be the difference between the adjacent bonds.

The Hamiltonian acts analogously to the $\mathbb{Z}_{2}$ case: i) a local term ensures that the four sites in $(7.10)$ multiply to the identity; ii) a plaquette term ensures the same for the corresponding sites of four tensors, and iii) a two-body term enforces equal weight for all bond configurations by applying $U_{s}$ coherently to the four adjacent sites.

Alternatively, we can again use the representation

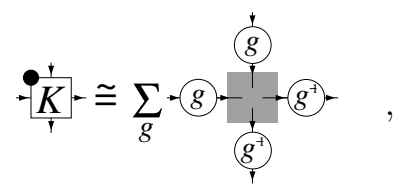

where the role of the Hamiltonian terms in this representation are to ensure that i) all $U_{g}$-invariant local configurations have equal weight, ii) the product of the $g$ 's around a plaquette is the identity, and iii) for any bond, all twirls appear with equal weight.

\subsection{Reducing the bond dimension: semi-regular represen- tations}

In the following, we would like to go beyond regular representations, and show how a PEPS which is $G$-injective with respect to a semi-regular representation can implement topological states which are equivalent to the quantum doubles, but with a lower bond dimension.

To this end, consider the tensor $K$ in the representation (7.11). Here, $g$ is the left-regular representation of a group, and can thus - by a proper relabelling

\footnotetext{
10 The PEPS representation depends on the rotation direction prescribed by the arrows around the plaquette. Eq. 7.10 is for clockwise rotation.
} 
of the virtual indices - be chosen to be of the form

$$
L_{g}=\bigoplus_{i=1}^{I} D^{i}(g) \otimes \mathbb{1}_{d_{i}} .
$$

Here, $D^{i}, i=1, \ldots, I$, are the irreducible representations of $g$, with dimensions $d_{i}$. Let us now take the smallest semi-regular representation

$$
V_{g}=\bigoplus_{i=1}^{I} D^{i}(g)
$$

of $G$, which contains every irreducible representation with multiplicity one, and define

$$
\Theta=\bigoplus_{i=1}^{I} \sqrt[4]{d_{i}} \mathbb{1}_{d_{i}} .
$$

[Note that $\Theta^{4}=\Delta$, with $\Delta$ from 4.6 .] With this, we define a tensor

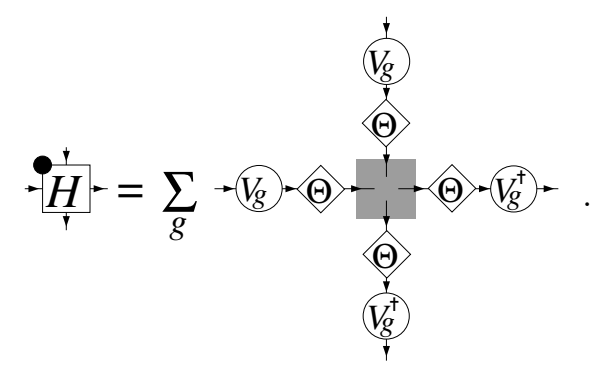

We now claim that the state given by the tensor $H$ is the same as the double model for the group $G$, i.e., the state described by (7.11) with the regular representation, up to unitaries between neighboring tensors which act on disjoint subsystems, i.e., local operations. This demonstrates that the double model has unneeded local degrees of freedom, and the bond dimension needed to represent the model is $\sum_{i} d_{i}$ rather than $\sum_{i} d_{i}^{2}$. Note that this is different from the bond dimension gained by renormalization in that no blocking of tensors is required.

In order to prove local equivalence of the two states, we will explicitly construct an isometric transformation which maps between the two states. (It has to be isometric as the dimension of the underlying space changes.) The transformation will act on the two endpoints of a bond simulateously, where it needs to implement a mapping between

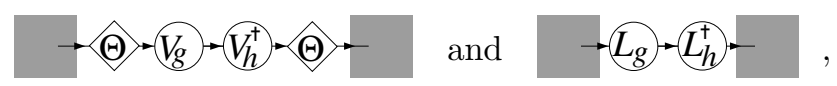

this is, between

$$
\sum_{i=1}^{I} \sum_{k_{i}, l_{i}=1}^{d_{i}} \sqrt{d_{i}} D_{k_{i}, l_{i}}^{i}\left(g h^{-1}\right)\left|i ; k_{i}\right\rangle\left\langle i ; l_{i}\right|
$$


and

$$
\sum_{i=1}^{I} \sum_{k_{i}, l_{i}=1}^{d_{i}} \sum_{m_{i}=1}^{d_{i}} D_{k_{i}, l_{i}}^{i}\left(g h^{-1}\right)\left|i ; k_{i} ; m_{i}\right\rangle\left\langle i ; l_{i} ; m_{i}\right| .
$$

This, however, can be accomplished by mapping between the basis elements of the two spaces as

$$
\left|i ; k_{i}\right\rangle\left\langle i ; l_{i}\left|\mapsto \frac{1}{\sqrt{d_{i}}} \sum_{m_{i}=1}^{d_{i}}\right| i ; k_{i} ; m_{i}\right\rangle\left\langle i ; l_{i} ; m_{i}\right| ;
$$

and thus, we have shown that the PEPS with tensor 7.12 , using a semi-regular representation, describes a state which is locally equivalent to the double model for the same group.

\section{Conclusions and outlook}

In this paper, we have presented a general framework for understanding Matrix Product States and Projected Entangled Pair states, and thus a large variety of interesting quantum states, in terms of symmetries. While this classification allowed us to reproduce the known results in one dimension, it also enabled us to go beyond one dimension and characterize the properties of two-dimensional systems with non-unique ground states. Using the symmetry-centered perspec-

tive, we could show how these states arise as finitely degenerate ground states of local Hamiltonians, and characterize the different ground states using symmetries at the closure. By looking only at these symmetries, we were able to explain the topological correction to the area law, the local indistinguishability of the ground state subspace, the anyonic nature of excitations of these systems, and why they are fixed points of renormalization group flows.

We believe that the framework set forward in this article will allow to explain a variety of things beyond what we have presented in this paper. For instance, the results on the parent Hamiltonian and ground state degeneracy can be directly extended to three and more spatial dimension (replacing the pair-conjugacy classes by triplet-conjugacy classes, etc.), as well as the statements made about the topological entropy, local indistinguishability, RG flows, and commuting Hamiltonians. By introducing excitations which are e.g. membranes of $g$ 's on the bonds, the framework of $G$-injective PEPS will also allow to explain the excitations of e.g. three-dimensional models.

The results presented here likely can be generalized to models with other symmetries. For instance, on might think of tensors for which the ordered product of the group elements assigned to all indices is the identity for all nonzero entries. This symmetry shares the crucial property of being stable under concatenation, and it allows for the definition of injective and isometric tensors, and thus should allow for comparable statements about topological entropy, local indistinguishability, renormalization transformations, or non-abelian excitations. Note that Kitaev's code state and the double models can be expressed 
in this form if one constructs the PEPS by starting from a ground state of $H_{B}$ and subsequently projects onto the ground state subspace of $H_{A}$. More generally, similar results might hold when considering more general symmetries, e.g. tensor categories used to define string-net models [61, or different tensornetwork based ansatzes [41,62. Note that from a different perspective, the role of so-called gauge-like symmetries for topological order has been studied in 63,64 .

The framework for understanding RG fixed points by mapping between isomorphic representations suggests the extension to the case of renormalization flows, e.g. by considering the tensor product of individual bond symmetries. For instance, one could use the fact that the normalized character of the tensor product of any faithful representation converges to the character of the regular representation, up to global phases, to show that RG flows converge towards $G$-isometric PEPS.

\section{Acknowledgements}

We would like to thank the Perimeter Institute for Theoretical Physics in Waterloo, Canada, where a major part of this work has been done, for its hospitality. We acknowledge helpful discussions with Miguel Aguado, Oliver Buerschaper, Sofyan Iblisdir, Frank Verstraete, and Michael Wolf. This work has been supported by the EU project QUEVADIS, the DFG Forschergruppe 635, the Gordon and Betty Moore Foundation through Caltech's Center for the Physics of Information, the National Science Foundation under Grant No. PHY-0803371, and the Spanish grants I-MATH, MTM2008-01366 and CCG08-UCM/ESP4394 .

\section{References}

[1] J. Eisert, M. Cramer, and M. Plenio, (2008), arXiv:0808.3773

[2] M. Hastings, J. Stat. Mech. , P08024 (2007), arXiv:0705.2024.

[3] L. Masanes, Phys. Rev. A 80, 052104 (2009), arXiv:0907.4672.

[4] M. B. Hastings, Phys. Rev. B 76, 035114 (2007), cond-mat/0701055.

[5] M. M. Wolf, F. Verstraete, M. B. Hastings, and J. I. Cirac, Phys. Rev. Lett. 100, 070502 (2008), arXiv.org:0704.3906.

[6] S. Iblisdir, D. Pérez-García, M. Aguado, and J. Pachos, Phys. Rev. B 79, 134303 (2009), arXiv:0806.1853.

[7] S. Östlund and S. Rommer, Phys. Rev. Lett. 75, 3537 (1995).

[8] F. Verstraete and J. I. Cirac, (2004), cond-mat/0407066 
[9] G. Vidal, Phys. Rev. Lett. 101, 110501 (2008), quant-ph/0610099.

[10] F. Verstraete, V. Murg, and J. I. Cirac, Advances in Physics 57, 143 (2008).

[11] M. Fannes, B. Nachtergaele, and R. F. Werner, Commun. Math. Phys. 144, 443 (1992).

[12] A. Affleck, T. Kennedy, E. H. Lieb, and H. Tasaki, Commun. Math. Phys. 115, 477 (1988).

[13] D. Perez-Garcia, F. Verstraete, M. M. Wolf, and J. I. Cirac, Quant. Inf. Comput. 7, 401 (2007), quant-ph/0608197.

[14] S. R. White, Phys. Rev. Lett. 69, 2863 (1992).

[15] F. Verstraete, D. Porras, and J. I. Cirac, Phys. Rev. Lett. 93, 227205 (2004), cond-mat/0404706.

[16] T. J. Osborne, Phys. Rev. A 75, 042306 (2007), quant-ph/0603137.

[17] F. Verstraete and J. I. Cirac, Phys. Rev. B 73, 094423 (2006), condmat/0505140.

[18] N. Schuch and J. I. Cirac, (2009), arXiv.org:0910.4264.

[19] D. Aharonov, I. Arad, and S. Irani, (2009), arXiv.org:0910.5055.

[20] N. Schuch, I. Cirac, and F. Verstraete, Phys. Rev. Lett. 100, 250501 (2008), arXiv:0802.3351

[21] D. Aharonov, D. Gottesman, S. Irani, and J. Kempe, Commun. Math. Phys. 287, 41 (2009), arXiv.org:0705.4077.

[22] F. Verstraete, M. A. Martin-Delgado, and J. I. Cirac, Phys. Rev. Lett. 92, 087201 (2004), quant-ph/0311087.

[23] L. C. Venuti and M. Roncaglia, Phys. Rev. Lett. 94, 207207 (2005), condmat/0503021.

[24] D. Perez-Garcia, M. Wolf, M. Sanz, F. Verstraete, and J. Cirac, Phys. Rev. Lett. 100, 167202 (2008), arXiv:0802.0447.

[25] S. Singh, H. Q. Zhou, and G. Vidal, (2007), cond-mat/0701427.

[26] F. Anfuso and A. Rosch, Phys. Rev. B 76, 085124 (2007), condmat/0702064

[27] M. Sanz, M. M. Wolf, D. Perez-Garcia, and J. I. Cirac, Phys. Rev. A 79, 042308 (2009), arXiv:0901.2223

[28] V. Karimipour and L. Memarzadeh, Phys. Rev. B 77, 094416 (2008), arXiv.org:0712.2018. 
[29] H.-H. Tu, G.-M. Zhang, and T. Xiang, Phys. Rev. B 78, 094404 (2008), arXiv:0806.1839.

[30] F. Pollmann, E. Berg, A. M. Turner, and M. Oshikawa, (2009), arXiv.org:0909.4059.

[31] M. M. Wolf, G. Ortiz, F. Verstraete, and J. I. Cirac, Phys. Rev. Lett. 97, 110403 (2006), cond-mat/0512180.

[32] M. Cozzini, R. Ionicioiu, and P. Zanardi, Phys. Rev. B 76, 104420 (2007), cond-mat/0611727.

[33] T.-C. Wei, (2008), arXiv.org:0810.2564.

[34] R. Orus, Phys. Rev. Lett. 100, 130502 (2008), arXiv:0711.2556

[35] C. Schön, E. Solano, F. Verstraete, J. I. Cirac, and M. M. Wolf, Phys. Rev. Lett. 95, 110503 (2005), quant-ph/0501096.

[36] L. Lamata, J. Leon, D. Perez-Garcia, D. Salgado, and E. Solano, Phys. Rev. Lett. 101, 180506 (2008), arXiv.org:0711.3652.

[37] F. Verstraete, J. Cirac, J. Latorre, E. Rico, and M. Wolf, Phys. Rev. Lett. 94, 140601 (2005), quant-ph/0410227.

[38] G. Vidal, Phys. Rev. Lett. 91, 147902 (2003), quant-ph/0301063.

[39] S. Iblisdir, R. Orus, and J. I. Latorre, Phys. Rev. B 75, 104305 (2007), cond-mat/0610530.

[40] J. I. Latorre, (2005), quant-ph/0510031.

[41] G. Sierra and M. A. Martin-Delgado, The density matrix renormalization group, quantum groups and conformal field theory, in Proceedings of the Workshop on the Exact Renormalization Group, Faro (Portugal), 1998, cond-mat/9811170

[42] M. B. Hastings, Phys. Rev. B 73, 085115 (2006), cond-mat/0508554.

[43] N. Schuch, M. M. Wolf, F. Verstraete, and J. I. Cirac, Phys. Rev. Lett. 98, 140506 (2007), quant-ph/0611050.

[44] F. Verstraete, M. M. Wolf, D. Perez-Garcia, and J. I. Cirac, Phys. Rev. Lett. 96, 220601 (2006), quant-ph/0601075.

[45] F. Verstraete and J. I. Cirac, Phys. Rev. A 70, 060302 (2004), quant$\mathrm{ph} / 0311130$

[46] R. Raussendorf and H. J. Briegel, Phys. Rev. Lett. 86, 5188 (2001), quant$\mathrm{ph} / 0010033$. 
[47] D. Gross and J. Eisert, Phys. Rev. Lett. 98, 220503 (2007), quant$\mathrm{ph} / 0609149$.

[48] D. Gross, J. Eisert, N. Schuch, and D. Perez-Garcia, Phys. Rev. A 76, 52315 (2007), arXiv:0706.3401.

[49] G. K. Brennen and A. Miyake, Phys. Rev. Lett. 101, 010502 (2008), arXiv:0803.1478.

[50] O. Buerschaper, M. Aguado, and G. Vidal, Phys. Rev. B 79, 085119 (2009), arXiv:0809.2393

[51] D. Perez-Garcia, F. Verstraete, J. I. Cirac, and M. M. Wolf, Quantum Inf.Comp. 8, 0650 (2008), arXiv:0707.2260.

[52] D. Perez-Garcia, M. Sanz, C. E. Gonzalez-Guillen, M. M. Wolf, and J. I. Cirac, (2009), arXiv.org:0908.1674.

[53] M. B. Hastings, Phys. Rev. B 69, 104431 (2004), cond-mat/0305505.

[54] S. Singh, R. N. C. Pfeifer, and G. Vidal, (2009), arXiv.org:0907.2994.

[55] M. Sanz, D. Perez-Garcia, M. M. Wolf, and J. I. Cirac, (2009), $\operatorname{arXiv/0909.5347.~}$

[56] A. Kitaev, Ann. Phys. 303, 2 (2003), quant-ph/9707021.

[57] J. Preskill, Lecture Notes for Physics 229: Quantum Information and Computation (, 1998), available at http://www.theory.caltech.edu/people/preskill/ph229.

[58] A. Kitaev and J. Preskill, Phys. Rev. Lett. 96, 110404 (2006), hepth/0510092.

[59] M. Levin and X.-G. Wen, Phys. Rev. Lett. 96, 110405 (2006), condmat/0510613

[60] S. T. Flammia, A. Hamma, T. L. Hughes, and X.-G. Wen, Phys. Rev. Lett. 103, 261601 (2009), arXiv:0909.3305.

[61] M. A. Levin and X.-G. Wen, Phys. Rev. B 71, 045110 (2005), condmat/0404617

[62] N. Maeshima, Y. Hieida, Y. Akutsu, T. Nishino, K. Okunishi, Phys. Rev. E 64 (2001) 016705; Y. Nishio, N. Maeshima, A. Gendiar, T. Nishino, cond-mat/0401115 (2004).

[63] Z. Nussinov and G. Ortiz, PNAS 106, 16944 (2009), cond-mat/0605316

[64] Z. Nussinov and G. Ortiz, Ann. Phys. 324, 977 (2009), cond-mat/0702377 